\title{
Enantioselective Total Synthesis of (+)-Euphorikanin A
}

Moritz J. Classen, Markus N. A. Böcker, Remo Roth, Willi M. Amberg, Erick M. Carreira

<erickm.carreira@org.chem.ethz.ch>

Eidgenössische Technische Hochschule Zürich, Vladimir-Prelog-Weg 3,

$\mathrm{HCl}, 8093$ Zürich, Switzerland

\section{Table of Contents}

General Methods

Experimental Procedures and Spectral Data

NMR Sprectra

Comparison of Natural and Synthetic (+)-Euphorikanin A

S42

Sources 


\subsection{General Methods}

Unless stated otherwise, reactions were performed in dry solvents and under an atmosphere of nitrogen. Dry solvents were either purchased from commercial sources, or for larger quantities obtained from a purification column composed of activated alumina.

Generally, chemicals were purchased from commercial sources (Acros, Alfa Aesar, Fluka, TCl) and used without further purification. Martins Sulfurane was stored and weighed in a glove box to avoid contact to air and moisture. Sml $\mathrm{Smas}_{2}$ prepared according to the literature procedure of Kagan and co-workers. ${ }^{1}$ However, if reactions were performed with a commercial solution of $\mathrm{Sml}_{2}$ purchased from Sigma-Aldrich, the same yields were achieved.

Aldehyde $\mathbf{8}$ was prepared according to a literature procedure from Altmann and co-workers, from commercial $(R)$-Roche ester. ${ }^{2}$

NMR spectra were recorded on Bruker DRX and Avance $(400 \mathrm{MHz})$ or DRXII $(500 \mathrm{MHz})$ spectrometers at room temperature. Signals are reported relative to the residual signal of the undeuterated solvent. Data are reported as follows: chemical shift, multiplicity ( $s=$ singlet, br $s$ = broad singlet, $\mathrm{d}$ = doublet, $\mathrm{t}=$ triplet, $\mathrm{q}=$ quartet, quin = quintet, $\mathrm{dd}=$ doublet of doublets, etc.) and integration.

Flash column chromatography was performed manually using glass columns with SigmaAldrich, $60 \AA$, 230-400 mesh silica gel using laboratory grade solvents. For small scale column chromatography, $\mathrm{Et}_{2} \mathrm{O}$ was distilled prior to use to remove $\mathrm{BHT}$, and stored under Argon in a brown glass bottle, while regularly being checked for peroxide formation

Thin layer chromatography was performed on glass plates pre-coated with silica gel (Merck, Silica gel $\left.60 \mathrm{~F}_{254}\right)$. Compounds were visualized using UV light and/or standard staining techniques involving $\mathrm{KMnO}_{4}$ or Seebach stain.

Infrared spectra (IR) were recorded on a Perkin Elmer Spectrum Two FT-IR (UATR) instrument as thin films and are reported as wavenumbers $\left(\mathrm{cm}^{-1}\right)$.

Optical rotation: Optical rotations were measured on a Jasco P-2000 Polarimeter, $10 \mathrm{~cm}$, $1.5 \mathrm{~mL}$ cell.

High resolution mass spectra (HRMS) were recorded on a Bruker Daltonics maXis ESI-QTOF by the ETH Zurich MS service. 


\section{Supporting Information}

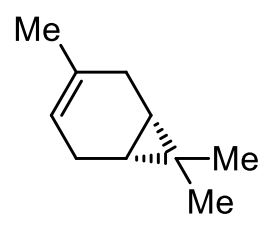

$(+)-3-$ carene (2)
$\mathrm{O}_{3}$

$\mathrm{MeOH}-\mathrm{CH}_{2} \mathrm{Cl}_{2}(90: 10),-78{ }^{\circ} \mathrm{C}, 2 \mathrm{~h}$ then $\mathrm{Me}_{2} \mathrm{~S}(1.8 \mathrm{eq})$ $-78^{\circ} \mathrm{C}$ to r.t., $12 \mathrm{~h}$

then $(\mathrm{MeO})_{3} \mathrm{CH}(4 \mathrm{eq}), \mathrm{CeCl}_{3} \cdot 7 \mathrm{H}_{2} \mathrm{O}(0.6 \mathrm{eq})$ r.t., $3.5 \mathrm{~h}$

A nitrogen purged solution of (1S)-(+)-3-carene (2) $(50 \mathrm{~mL}, 320 \mathrm{mmol}, 90 \%$ purity, 1.0 eq) in $\mathrm{MeOH}-$ $\mathrm{CH}_{2} \mathrm{Cl}_{2}(90: 10,300 \mathrm{~mL})$ at $-78{ }^{\circ} \mathrm{C}$ was treated with ozone for 2 hours. After purging with nitrogen, dimethyl sulfide ( $42 \mathrm{~mL}, 570 \mathrm{mmol}, 1.8 \mathrm{eq}$ ) was added and the reaction mixture was stirred for 12 hours while slowly warming up to room temperature. After addition of trimethoxymethane $(150 \mathrm{~mL}$, $1.4 \mathrm{~mol}, 4.3 \mathrm{eq})$ and cerium(III) chloride heptahydrate $(66 \mathrm{~g}, 180 \mathrm{mmol}, 0.60 \mathrm{eq})$ the reaction was stirred at room temperature for 3.5 hours and concentrated in vacuo. The concentrate was dissolved in saturated aqueous $\mathrm{NaHCO}_{3}(250 \mathrm{~mL})$, extracted with ether $(3 \times 500 \mathrm{~mL})$, dried over $\mathrm{Na}_{2} \mathrm{SO}_{4}$ and concentrated in vacuo. The resulting yellow oil was used in the following step without further purification. 


\section{Supporting Information}

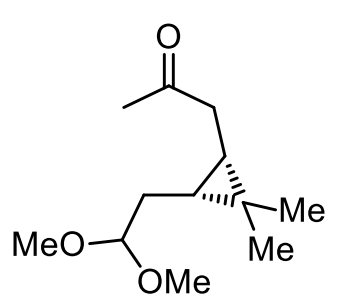

SI-1

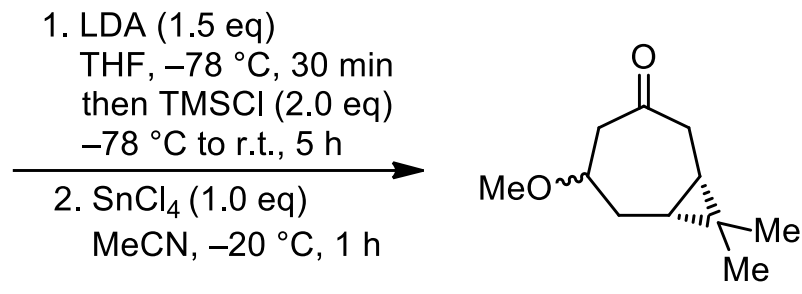

SI-2

To a solution of freshly prepared $0.5 \mathrm{M}$ solution of LDA in THF $(0.60 \mathrm{~L}, 0.30 \mathrm{~mol}, 1.4 \mathrm{eq})$ at $-78^{\circ} \mathrm{C}$ was slowly added a solution of SI-1 $(49.0 \mathrm{~g}, 220 \mathrm{mmol}, 1.0 \mathrm{eq})$ in THF $(50 \mathrm{~mL})$ over 30 minutes and the reaction was stirred for 30 minutes before adding freshly distilled TMSCl ( $57 \mathrm{~mL}, 450 \mathrm{mmol}, 2.0$ eq) and stirring for an additional 5 hours while warming to room temperature. After diluting with hexane $(500 \mathrm{~mL})$ the reaction mixture was quenched with saturated aqueous $\mathrm{NaHCO}_{3}$ solution $(500 \mathrm{~mL})$, extracted with hexane $(3 \times 800 \mathrm{~mL})$, dried over $\mathrm{Na}_{2} \mathrm{SO}_{4}$ and concentrated in vacuo.

To a well stirred solution of tin(IV) chloride $(27.0 \mathrm{~mL}, 231 \mathrm{mmol}, 1.0 \mathrm{eq})$ in $\mathrm{MeCN}(50 \mathrm{~mL})$ at $-20^{\circ} \mathrm{C}$ was added dropwise a solution of the crude enol ether in MeCN $(450 \mathrm{~mL})$ over 1 hour. After stirring for 5 minutes the reaction was diluted with diethyl ether $(200 \mathrm{~mL})$, quenched with saturated aqueous $\mathrm{NaHCO}_{3}(1.5 \mathrm{~L})$, extracted with ether $(6 \times 2 \mathrm{~L})$, dried over $\mathrm{Na}_{2} \mathrm{SO}_{4}$ and concentrated in vacuo. Flash column chromatography (hexane/ $\left.\mathrm{Et}_{2} \mathrm{O}, 93: 7\right)$ provided the desired product $\mathbf{S I - 2}$ (13 g, $72 \mathrm{mmol}, 33 \%$ ) in an inconsequential mixture of diastereomers (anti-syn 2:1) as a colorless oil.

The analytical data matched previous reports

${ }^{1} \mathrm{H}-\mathrm{NMR}$ : (400 MHz, Chloroform-d) $83.71-3.67(\mathrm{~m}, 2 \mathrm{H}), 3.65-3.58(\mathrm{~m}, 1 \mathrm{H}), 3.38(\mathrm{~s}, 6 \mathrm{H}), 3.36(\mathrm{~s}, 3 \mathrm{H})$, $3.12-3.04(\mathrm{~m}, 3 \mathrm{H}), 2.72-2.65(\mathrm{~m}, 2 \mathrm{H}), 2.61-2.54(\mathrm{~m}, 3 \mathrm{H}), 2.48(\mathrm{dd}, J=11.9,7.1 \mathrm{~Hz}, 1 \mathrm{H}), 2.34$ (ddd, $J=14.4,9.2,2.8 \mathrm{~Hz}, 3 \mathrm{H}), 2.21-2.12(\mathrm{~m}, 3 \mathrm{H}), 1.33-1.25(\mathrm{~m}, 1 \mathrm{H}), 1.13(\mathrm{~s}, 7 \mathrm{H}), 1.10(\mathrm{~s}, 3 \mathrm{H}), 1.08(\mathrm{~s}, 3 \mathrm{H})$, $1.03(\mathrm{~s}, 6 \mathrm{H}), 1.01-0.96(\mathrm{~m}, 2 \mathrm{H}), 0.85-0.78(\mathrm{~m}, 2 \mathrm{H}), 0.72(\mathrm{dtd}, J=8.0,3.0,1.8 \mathrm{~Hz}, 2 \mathrm{H})$.

${ }^{13}$ C-NMR: (101 MHz, Chloroform-d) $\delta$ 209.8, 209.7, 78.6, 76.8, 56.6, 56.5, 49.3, 48.2, 39.7, 39.1, 29.9, $28.6,28.5,27.1,22.7,22.0,21.2,20.2,20.1,15.2,15.2$. 


\section{Supporting Information}

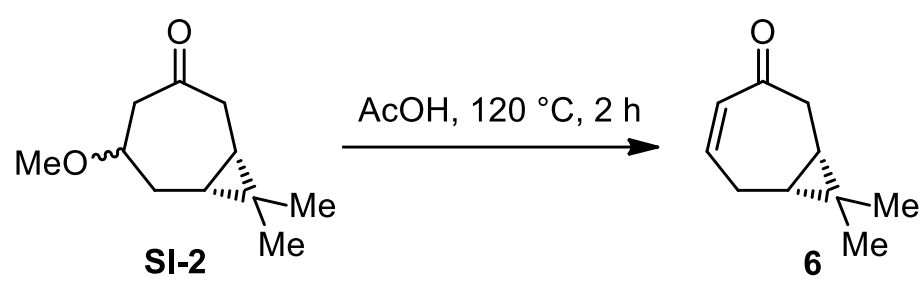

A well stirred solution of SI-2 (13 g, $72 \mathrm{mmol}, 1.0 \mathrm{eq})$ in acetic acid $(0.30 \mathrm{~L})$ was refluxed for 2 hours, quenched with saturated aqueous $\mathrm{Na}_{2} \mathrm{CO}_{3}$, extracted with ether, dried over $\mathrm{Na}_{2} \mathrm{SO}_{4}$. After careful concentration in vacuo, column chromatography (hexane- $\mathrm{Et}_{2} \mathrm{O}, 97: 3$ ) yielded the desired product 6 $(8.1 \mathrm{~g}, 43 \mathrm{mmol}, 59 \%)$ as a pale-yellow oil that solidified in the freezer.

The analytical data is in agreement to previous reports of Yamaka ${ }^{3}$ and co-workers and Wood ${ }^{4}$ and coworkers.

${ }^{1} \mathrm{H}-\mathrm{NMR}(400 \mathrm{MHz}$, Chloroform-d) $\delta 6.81$ (ddd, $J=11.6,8.7,3.8 \mathrm{~Hz}, 1 \mathrm{H}$ ), 5.96 (ddd, $J=11.6,3.1,2.2 \mathrm{~Hz}$, 1H), 2.74 (ddd, $J=13.7,5.0,2.2 \mathrm{~Hz}, 1 \mathrm{H}$ ), 2.56 (ddd, $J=16.4,8.7,6.1 \mathrm{~Hz}, 1 \mathrm{H}), 2.36-2.26(\mathrm{~m}, 1 \mathrm{H}), 2.14$ $-2.03(\mathrm{~m}, 1 \mathrm{H}), 1.21-1.13(\mathrm{~m}, 4 \mathrm{H}), 1.08(\mathrm{~s}, 3 \mathrm{H}), 0.71(\mathrm{ddd}, J=12.0,9.1,5.0 \mathrm{~Hz}, 1 \mathrm{H})$.

${ }^{13}$ C-NMR (101 MHz, Chloroform-d) $\delta$ 201.7, 148.5, 133.8, 42.1, 31.4, 28.6, 25.8, 24.6, 23.0, 15.7. 

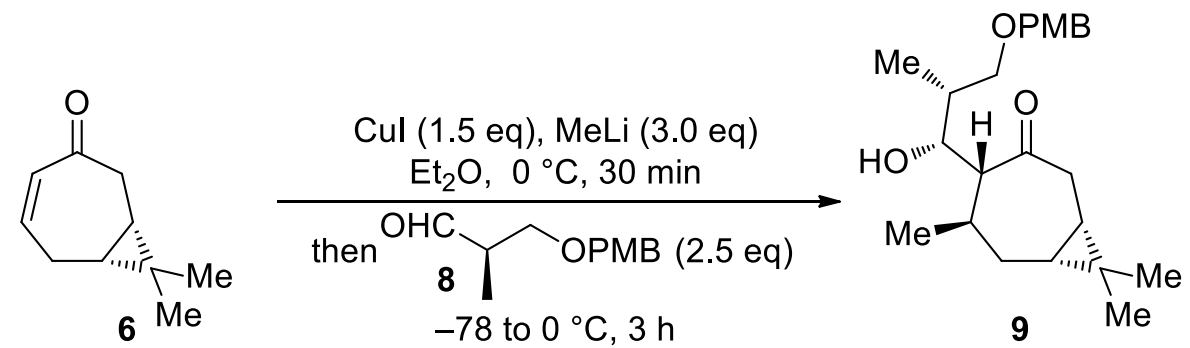

To a well stirred solution of copper $(\mathrm{l})$ iodide $(4.8 \mathrm{~g}, 25 \mathrm{mmol}, 1.5 \mathrm{eq})$ in diethyl ether $(130 \mathrm{~mL})$ at $0{ }^{\circ} \mathrm{C}$ was added a $1.6 \mathrm{M}$ solution of methyl lithium in diethyl ether $(32 \mathrm{~mL}, 0.050 \mathrm{~mol}, 3.0 \mathrm{eq})$ and after stirring for 5 minutes the mixture was cooled to $-78^{\circ} \mathrm{C}$. At $-78^{\circ} \mathrm{C} 6(2.5 \mathrm{~g}, 17 \mathrm{mmol}, 1.0 \mathrm{eq})$ was added and the reaction was stirred for 30 minutes until completion was confirmed by TLC. 8 ( $8.7 \mathrm{~g}, 42 \mathrm{mmol}$, $2.5 \mathrm{eq})$ in diethyl ether $(3 \times 7 \mathrm{~mL})$ was added over 10 minutes and the reaction was let warm to $0{ }^{\circ} \mathrm{C}$ over 3 hours. After quenching with saturated aqueous $\mathrm{NaHCO}_{3}(250 \mathrm{~mL})$, extracting with diethyl ether $\left(3 \times 250 \mathrm{~mL}\right.$ ), drying over $\mathrm{Na}_{2} \mathrm{SO}_{4}$, celite filtration and purification via column chromatography (hexane$\mathrm{Et}_{2} \mathrm{O}, 95: 5$ to $\left.80: 20\right)$ the desired diastereomer $9(4.9 \mathrm{~g}, 13 \mathrm{mmol}, 79 \%)$ was isolated as a yellow oil.

${ }^{1} \mathrm{H}-\mathrm{NMR}\left(400 \mathrm{MHz}, \mathrm{CDCl}_{3}\right): \delta 7.25(\mathrm{~d}, J=8.7 \mathrm{~Hz}, 2 \mathrm{H}), 6.87(\mathrm{~d}, J=8.7 \mathrm{~Hz}, 2 \mathrm{H}), 4.42(\mathrm{~s}, 2 \mathrm{H}), 3.80(\mathrm{~s}, 3 \mathrm{H})$, $3.60(\mathrm{dd}, J=9.1,4.5 \mathrm{~Hz}, 1 \mathrm{H}$ ), $3.56-3.51(\mathrm{~m}, 1 \mathrm{H}), 3.49-3.40(\mathrm{~m}, 1 \mathrm{H}), 2.97$ (ddd, $J=8.7,2.9,0.9 \mathrm{~Hz}$, $1 \mathrm{H}), 2.68(\mathrm{dd}, J=17.8,9.9 \mathrm{~Hz}, 1 \mathrm{H}), 2.36-2.25(\mathrm{~m}, 1 \mathrm{H}), 2.20$ (ddd, $J=17.8,6.7,1.1 \mathrm{~Hz}, 1 \mathrm{H}$ ), $1.89-$ $1.78(\mathrm{~m}, 1 \mathrm{H}), 1.75-1.68(\mathrm{~m}, 1 \mathrm{H}), 1.13(\mathrm{~d}, J=6.8 \mathrm{~Hz}, 3 \mathrm{H}), 1.08(\mathrm{~s}, 3 \mathrm{H}), 1.07-0.99(\mathrm{~m}, 1 \mathrm{H}), 0.95-0.87$ $(\mathrm{m}, 7 \mathrm{H}), 0.76$ (ddd, $J=9.9,8.7,6.7 \mathrm{~Hz}, 1 \mathrm{H})$.

${ }^{13} \mathrm{C}-\mathrm{NMR}\left(101 \mathrm{MHz}, \mathrm{CDCl}_{3}\right)$ : $\delta$ 218.9, 159.1, 130.6, 129.2, 113.7, 73.5, 72.8, 72.6, 55.3, 55.1, 40.8, $38.7,32.0,28.6,28.0,26.9,22.1,20.0,19.1,18.8,15.2,15.1$.

IR (thin film, cm ${ }^{-1}$ ): 3479, 2957, 2934, 2868, 2867, 1685, 1612, 1586, 1513, 1458, 1418, 1417, 1377, $1302,1246,1173,1137,1083,1035,986,957,909,819,757,708,687,638,563,516$.

HRMS (ESI) $m / z$ : exact mass calculated for $\mathrm{C}_{23} \mathrm{H}_{34} \mathrm{NaO}_{4}[\mathrm{M}+\mathrm{Na}]^{+}, 397.2349$; found: 397.2347. []$_{D}{ }^{25^{\circ}}=+89.7\left(\mathrm{c}=0.5, \mathrm{CHCl}_{3}\right)$. 


\section{Supporting Information}
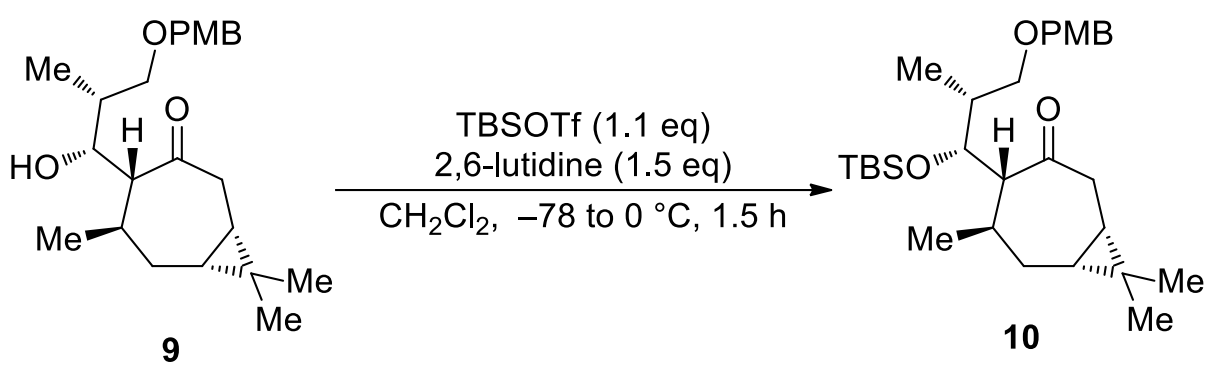

To a solution of $9(4.6 \mathrm{~g}, 12 \mathrm{mmol}, 1.0 \mathrm{eq})$ in $\mathrm{CH}_{2} \mathrm{Cl}_{2}(125 \mathrm{~mL})$ at $-78{ }^{\circ} \mathrm{C}$ was added 2,6-lutidine $(2.2 \mathrm{~mL}$, $19 \mathrm{mmol}, 1.5 \mathrm{eq})$. After stirring for 30 seconds TBSOTf ( $3.0 \mathrm{~mL}, 13 \mathrm{mmol}, 1.1 \mathrm{eq}$ ) was added and the solution was warmed to RT over 1.5 hours. The mixture was quenched with saturated aqueous $\mathrm{NH}_{4} \mathrm{Cl}$ $(100 \mathrm{~mL})$ and diluted with water $(200 \mathrm{~mL})$. After extracting with $\mathrm{CH}_{2} \mathrm{Cl}_{2}(4 \times 200 \mathrm{~mL})$ the combined organic phases were dried over $\mathrm{Na}_{2} \mathrm{SO}_{4}$, filtered, and concentrated in vacuo. Flash column chromatography (hexane- $\left.\mathrm{Et}_{2} \mathrm{O}, 95: 5\right)$ provided the desired product $10(4.7 \mathrm{~g}, 10 \mathrm{mmol}, 78 \%)$ as a pale oil.

${ }^{1} \mathrm{H}-\mathrm{NMR}\left(400 \mathrm{MHz}, \mathrm{CDCl}_{3}\right): \delta 7.24(\mathrm{~d}, J=8.7,2 \mathrm{H}), 6.87(\mathrm{~d}, J=8.7 \mathrm{~Hz}, 2 \mathrm{H}), 4.41(\mathrm{~s}, 2 \mathrm{H}), 4.11(\mathrm{dd}, J=8.1$, $2.9 \mathrm{~Hz}, 1 \mathrm{H}), 3.81(\mathrm{~s}, 3 \mathrm{H}), 3.80(\mathrm{~s}, 1 \mathrm{H}), 3.27(\mathrm{dd}, J=9.4,7.1 \mathrm{~Hz}, 1 \mathrm{H}), 2.64(\mathrm{dd}, J=8.2,4.0 \mathrm{~Hz}, 1 \mathrm{H}), 2.45$ (ddd, $J=12.9,7.6,1.3 \mathrm{~Hz}, 1 \mathrm{H}$ ), 2.29 (dd, $J=12.9,9.4 \mathrm{~Hz}, 1 \mathrm{H}$ ), 2.13 (qd, $J=7.1,2.8 \mathrm{~Hz}, 1 \mathrm{H}$ ), $2.01-1.92$ $(\mathrm{m}, 1 \mathrm{H}), 1.70(\mathrm{dt}, J=15.0,5.3 \mathrm{~Hz}, 1 \mathrm{H}), 1.43(\mathrm{~s}, 1 \mathrm{H}), 1.21(\mathrm{t}, J=7.0 \mathrm{~Hz}, 1 \mathrm{H}), 1.06(\mathrm{~s}, 3 \mathrm{H}), 1.02(\mathrm{~s}, 3 \mathrm{H})$, $1.00(\mathrm{~d}, J=7.0 \mathrm{~Hz}, 3 \mathrm{H}), 0.96(\mathrm{~d}, J=7.0 \mathrm{~Hz}, 3 \mathrm{H}), 0.90(\mathrm{~d}, J=15.2 \mathrm{~Hz}, 1 \mathrm{H}), 0.83(\mathrm{~s}, 9 \mathrm{H}), 0.08--0.02(\mathrm{~m}$, $6 \mathrm{H})$.

${ }^{13} \mathrm{C}-\mathrm{NMR}\left(101 \mathrm{MHz}, \mathrm{CDCl}_{3}\right): \delta 210.6,130.6,129.2,113.7,74.8,72.8,71.9,62.7,55.3,38.7,38.6,31.4$, 28.6, 26.1, 26.0, 23.0, 21.3, 20.0, 19.1, 18.4, 15.3, 13.6, -3.8, -4.2 .

IR (thin film, cm ${ }^{-1}$ ): 2954, 2930, 2857, 1704, 1613, 1587, 1513, 1463, 1378, 1362, 1302, 1248, 1173, $1085,1084,1062,1037,959,938,871,832,774,666,641,587,586,516$.

HRMS (ESI) $\mathrm{m} / \mathrm{z}$ : exact mass calculated for $\mathrm{C}_{29} \mathrm{H}_{48} \mathrm{NaO}_{4} \mathrm{Si}[\mathrm{M}+\mathrm{Na}]^{+}, 511.3214$; found: 511.3209 . $[\mathrm{a}]_{\mathrm{D}}^{25^{\circ}}=+70.1\left(\mathrm{c}=1, \mathrm{CHCl}_{3}\right)$. 


\section{Supporting Information}

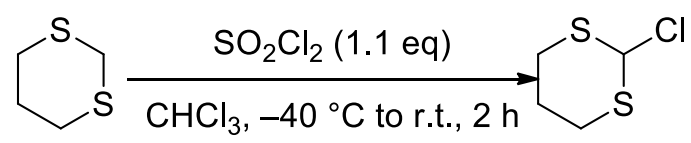

SI-3

11

To a well stirred solution of 1,3-dithiane $(1.0 \mathrm{~g}, 0.010 \mathrm{~mol}, 1.0 \mathrm{eq})$ in chloroform $(25 \mathrm{~mL})$ at $-40{ }^{\circ} \mathrm{C}$ was added dropwise sulfuryl chloride $(0.89 \mathrm{~mL}, 11 \mathrm{mmol}, 1.1 \mathrm{eq})$ in chloroform $(5 \mathrm{~mL})$ over 30 minutes. After stirring for an additional 30 minutes the solution was let warm to room temperature and stirred for $2 \mathrm{~h}$. The solution was then cooled to $0^{\circ} \mathrm{C}$ and concentrated in vacuo.

Due to the high sensitivity of the crude product towards water the crude product was never taken out of a $\mathrm{N}_{2}$ atmosphere. It was rather, dissolved in THF $(10 \mathrm{~mL})$, stirred at $0{ }^{\circ} \mathrm{C}$ for 1 hour and used as the crude solution. 


\section{Supporting Information}

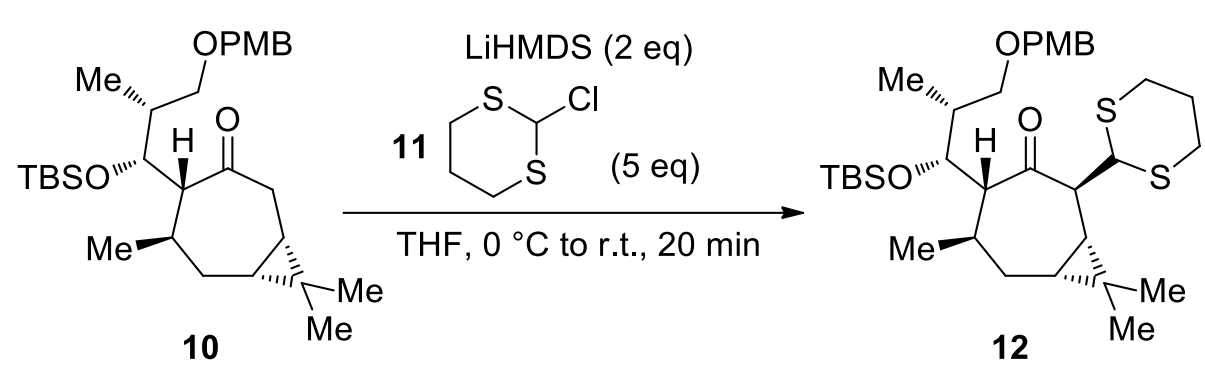

A solution of $10(0.610 \mathrm{~g}, 1.25 \mathrm{mmol}, 1.0 \mathrm{eq})$ in THF $(12.0 \mathrm{~mL})$ at $0{ }^{\circ} \mathrm{C}$ was treated with a $1 \mathrm{M}$ solution of LiHMDS in THF ( $2.50 \mathrm{~mL}, 2.50 \mathrm{mmol}, 2.0 \mathrm{eq}$ ) and stirred for $0.75 \mathrm{~h}$. Crude 11 as a an approximately $1 \mathrm{M}$ solution in THF $(5.00 \mathrm{~mL}, 5.00 \mathrm{mmol}, 4.0 \mathrm{eq})$ was added and the solution was warmed to room temperature. After stirring at room temperature for 20 minutes the reaction was quenched with sat. aq. $\mathrm{NH}_{4} \mathrm{Cl}(15 \mathrm{~mL})$, diluted with water $(15 \mathrm{~mL})$, extracted with diethyl ether $(3 \times 50 \mathrm{~mL})$, dried over $\mathrm{Na}_{2} \mathrm{SO}_{4}$ and concentrated in vacuo. Column chromatography (hexane-Et $2 \mathrm{O}, 90: 10$ ) yielded the product 12 (678 mg, $1.12 \mathrm{mmol}, 90 \%$ ) as a light-yellow oil.

${ }^{1}$ H-NMR: $\left(400 \mathrm{MHz}, \mathrm{CDCl}_{3}\right) \delta 7.24(\mathrm{~d}, J=8.6 \mathrm{~Hz}, 2 \mathrm{H}), 6.85(\mathrm{~d}, J=8.7 \mathrm{~Hz}, 2 \mathrm{H}), 4.62(\mathrm{~d}, J=6.3 \mathrm{~Hz}, 1 \mathrm{H})$, $4.46-4.34(\mathrm{~m}, 2 \mathrm{H}), 3.92(\mathrm{dd}, J=4.9,2.1 \mathrm{~Hz}, 1 \mathrm{H}), 3.80(\mathrm{~s}, 3 \mathrm{H}), 3.60(\mathrm{dd}, J=9.4,4.1 \mathrm{~Hz}, 1 \mathrm{H}), 3.27$ (dd, $J=9.4,8.3 \mathrm{~Hz}, 1 \mathrm{H}), 3.05-2.89(\mathrm{~m}, 2 \mathrm{H}), 2.92-2.85(\mathrm{~m}, 1 \mathrm{H}), 2.85-2.73(\mathrm{~m}, 2 \mathrm{H}), 2.55(\mathrm{dd}, J=9.3$, $6.3 \mathrm{~Hz}, 1 \mathrm{H}), 2.27(\mathrm{ddt}, J=8.6,6.8,4.5 \mathrm{~Hz}, 1 \mathrm{H}), 2.17-2.02(\mathrm{~m}, 2 \mathrm{H}), 1.91-1.75(\mathrm{~m}, 1 \mathrm{H}), 1.67-1.57(\mathrm{~m}$, $1 \mathrm{H}), 1.25-1.16(\mathrm{~m}, 1 \mathrm{H}), 1.15-1.08(\mathrm{~m}, 6 \mathrm{H}), 1.07(\mathrm{~d}, J=6.8 \mathrm{~Hz}, 3 \mathrm{H}), 1.01(\mathrm{~s}, 3 \mathrm{H}), 0.89(\mathrm{~s}, 9 \mathrm{H}), 0.87(\mathrm{~s}$, $1 \mathrm{H}), 0.76(\mathrm{t}, J=9.1 \mathrm{~Hz}, 1 \mathrm{H}), 0.11(\mathrm{~s}, 3 \mathrm{H}), 0.04(\mathrm{~s}, 3 \mathrm{H})$.

${ }^{13}$ C-NMR: $\left(101 \mathrm{MHz}, \mathrm{CDCl}_{3}\right) \delta 212.5,159.0,131.0,129.2,113.7,76.2,72.6,72.5,61.4,55.3,53.3$, 51.6, 37.6, 33.2, 31.1, 30.9, 29.6, 28.2, 26.2, 25.7, 24.2, 22.1, 21.0, 20.6, 18.4, 16.7, 15.8, -3.2, -4.7. IR (thin film, $\mathrm{cm}^{-1}$ ): 2953, 2928, 2857, 1698, 1612, 1586, 1513, 1463, 1422, 1378, 1361, 1302, 1275, $1248,1172,1087,1039,1005,903,869,834,775,704,669,639,587,514,432$.

HRMS (ESI): $m / z$ : exact mass calculated for $\mathrm{C}_{33} \mathrm{H}_{55} \mathrm{O}_{4} \mathrm{~S}_{2} \mathrm{Si}[\mathrm{M}+\mathrm{H}]^{+}, 607.3306$; found: 607.3296. $[\mathrm{a}]_{\mathrm{D}} \mathrm{5}^{\circ}=169.3\left(\mathrm{c}=1.0, \mathrm{CHCl}_{3}\right)$ 


\section{Supporting Information}
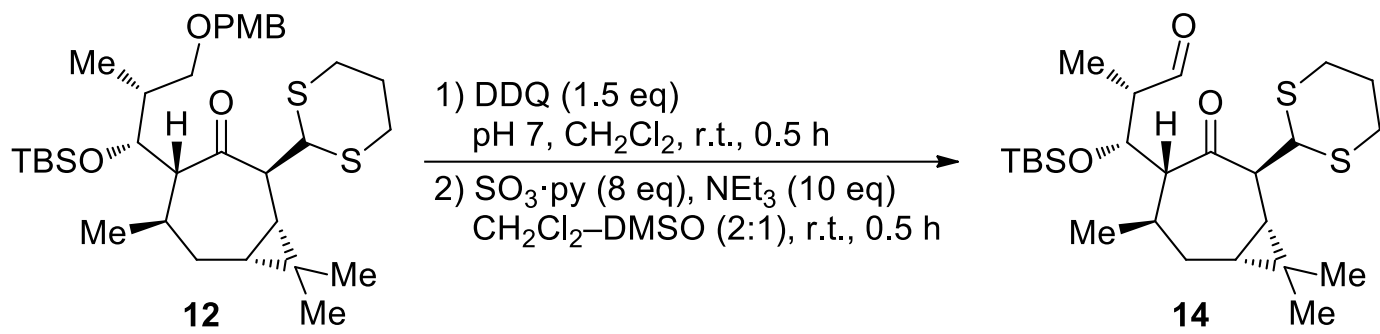

To a well stirred solution of 12 (58 mg, $0.097 \mathrm{mmol}, 1.0 \mathrm{eq})$ in $\mathrm{CH}_{2} \mathrm{Cl}_{2}$-aqueous $\mathrm{pH} 8$ buffer $(10: 1,1.1$ $\mathrm{mL}$ ) was added DDQ (38 $\mathrm{mg}, 0.17 \mathrm{mmol}, 1.8 \mathrm{eq})$, and the reaction was stirred at room temperature for $0.5 \mathrm{~h}$. After quenching with sat. aq. $\mathrm{NaHCO}_{3}(2 \mathrm{~mL})$, extracting with $\mathrm{CH}_{2} \mathrm{Cl}_{2}(4 \times 5 \mathrm{~mL})$, drying over $\mathrm{Na}_{2} \mathrm{SO}_{4}$, and concentrating in vacuo the crude product was redissolved in $\mathrm{CH}_{2} \mathrm{Cl}_{2}-\mathrm{DMSO}(2: 1,1 \mathrm{~mL})$. While constantly stirring at room temperature dry triethylamine $(0.13 \mathrm{~mL}, 1.0 \mathrm{mmol}, 10 \mathrm{eq})$ and $\mathrm{SO}_{3} \cdot \mathrm{py}$ $(0.12 \mathrm{~g}, 0.76 \mathrm{mg}, 8.0 \mathrm{mmol})$ were added consecutively. After stirring at room temperature for $0.5 \mathrm{~h}$ the reaction was quenched with sat. aq. $\mathrm{NaHCO}_{3}(2 \mathrm{~mL})$, extracted with diethyl ether $(3 \times 5 \mathrm{~mL})$, dried over $\mathrm{Na}_{2} \mathrm{SO}_{4}$, and concentrated in vacuo. Purification via column chromatography (hexane- $\mathrm{Et}_{2} \mathrm{O}, 90: 10$ ) yielded the product 14 as a colorless oil ( $21 \mathrm{mg}, 0.043 \mathrm{mmol}, 45 \%$ ).

${ }^{1} \mathrm{H}-\mathrm{NMR}\left(400 \mathrm{MHz}, \mathrm{CDCl}_{3}\right) \delta 9.86(\mathrm{~d}, J=3.5 \mathrm{~Hz}, 1 \mathrm{H}), 4.59(\mathrm{~d}, J=5.2 \mathrm{~Hz}, 1 \mathrm{H}), 4.13(\mathrm{t}, J=2.3 \mathrm{~Hz}, 1 \mathrm{H})$, 3.19 (ddd, $J=9.4,2.0,0.9 \mathrm{~Hz}, 1 \mathrm{H}$ ), $3.03-2.92(\mathrm{~m}, 3 \mathrm{H}), 2.81$ (ddd, $J=14.1,4.5,3.2 \mathrm{~Hz}, 2 \mathrm{H}$ ), 2.44 (dd, $J=8.5,5.2 \mathrm{~Hz}, 1 \mathrm{H}), 2.20(\mathrm{dtt}, J=9.4,4.6,2.4 \mathrm{~Hz}, 1 \mathrm{H}), 2.13-2.05(\mathrm{~m}, 1 \mathrm{H}), 1.85(\mathrm{dtt}, J=14.1,12.4$, $3.2 \mathrm{~Hz}, 1 \mathrm{H}), 1.69-1.56(\mathrm{~m}, 1 \mathrm{H}), 1.21(\mathrm{dd}, J=6.9,4.9 \mathrm{~Hz}, 6 \mathrm{H}), 1.16(\mathrm{~s}, 3 \mathrm{H}), 1.07-1.00(\mathrm{~m}, 2 \mathrm{H}), 0.97(\mathrm{~s}$, $3 \mathrm{H}), 0.95-0.92(\mathrm{~m}, 1 \mathrm{H}), 0.89(\mathrm{~s}, 9 \mathrm{H}), 0.16(\mathrm{~s}, 3 \mathrm{H}), 0.06(\mathrm{~s}, 3 \mathrm{H})$.

${ }^{13}$ C-NMR: $\left(101 \mathrm{MHz} \mathrm{CDCl}_{3}\right) \delta$ 214.3, 206.0, 76.0, 60.7, 54.0, 52.6, 49.9, 33.1, 31.1, 30.7, 30.1, 28.2, $25.9,25.4,23.2,21.9,20.8,20.3,18.1,15.6,14.4,3.4,5.2$.

IR (thin film, cm ${ }^{-1}$ ): 2954, 2929, 2899, 2858, 1720, 1698, 1463,1422, 1389, 1378, 1361, 1309, 1276, $1253,1223,1186,1144,1092,1034,1005,938,902,869,833,812,775,732,704,670,646,586,502$, $449,407$.

HRMS (ESI): $m / z$ : exact mass calculated for $\mathrm{C}_{25} \mathrm{H}_{44} \mathrm{NaO}_{3} \mathrm{~S}_{2} \mathrm{Si}[\mathrm{M}+\mathrm{Na}]^{+}$, 507.2393; found: 507.2396.

$[\mathrm{a}]_{\mathrm{D}}{ }^{25^{\circ}}=79.7\left(\mathrm{c}=0.75, \mathrm{CHCl}_{3}\right)$ 


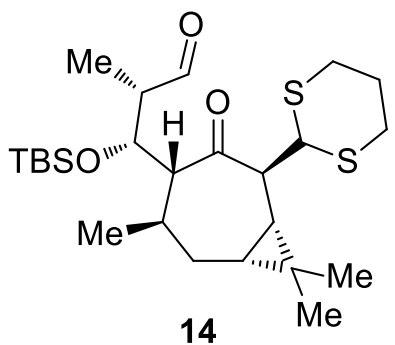

14

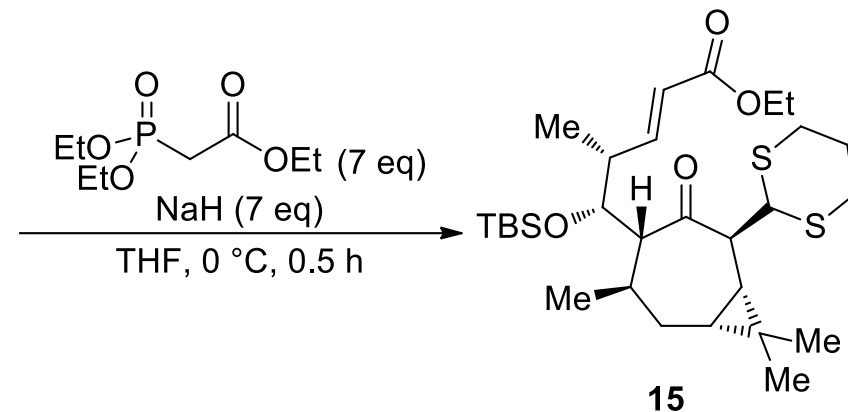

15

To a well stirred solution of $\mathrm{NaH}(7 \mathrm{mg}, 0.2 \mathrm{mmol}, 7$ eq) in THF $(0.3 \mathrm{~mL})$ was added ethyl 2(diethoxyphosphoryl)acetate $(0.03 \mathrm{~mL}, 0.2 \mathrm{mmol}, 7 \mathrm{eq})$ at $0{ }^{\circ} \mathrm{C}$. After $10 \mathrm{~min} 14$ (11 mg, $0.023 \mathrm{mmol}$, $1.0 \mathrm{eq})$ in THF $(0.3 \mathrm{~mL})$ was added. After stirring at $0{ }^{\circ} \mathrm{C}$ for $0.5 \mathrm{~h}$ the reaction was quenched with sat. aq. $\mathrm{NH}_{4} \mathrm{Cl}(2 \mathrm{~mL})$, diluted with water $(2 \mathrm{~mL})$, extracted with diethyl ether $(3 \times 5 \mathrm{~mL})$, dried over $\mathrm{Na}_{2} \mathrm{SO}_{4}$, and concentrated in vacuo. Column chromatography (hexane-Et $2 \mathrm{O}, 90: 10$ ) yielded the product 15 (8 $\mathrm{mg}, 0.02 \mathrm{mmol}, 65 \%)$ as a colorless oil.

${ }^{1} \mathrm{H}$-NMR: (500 MHz, Chloroform-d) $87.26-7.20(\mathrm{~m}, 1 \mathrm{H}), 5.79$ (dd, $\left.J=15.9,0.9 \mathrm{~Hz}, 1 \mathrm{H}\right), 4.61(\mathrm{~d}, J=5.8$ $\mathrm{Hz}, 1 \mathrm{H}), 4.22-4.11(\mathrm{~m}, 2 \mathrm{H}), 3.91(\mathrm{dd}, J=3.3,2.0 \mathrm{~Hz}, 1 \mathrm{H}), 3.11-2.92(\mathrm{~m}, 4 \mathrm{H}), 2.84-2.79(\mathrm{~m}, 2 \mathrm{H}), 2.47$ (dd, $J=9.0,5.7 \mathrm{~Hz}, 1 \mathrm{H}$ ), 2.17 (dddd, $J=9.3,6.8,4.6,2.9 \mathrm{~Hz}, 1 \mathrm{H}$ ), $2.12-2.07(\mathrm{~m}, 1 \mathrm{H}), 1.90-1.81(\mathrm{~m}$, $1 \mathrm{H}), 1.56(\mathrm{~s}, 1 \mathrm{H}), 1.27(\mathrm{t}, J=7.1 \mathrm{~Hz}, 4 \mathrm{H}), 1.14-1.12(\mathrm{~m}, 6 \mathrm{H}), 0.98(\mathrm{~s}, 3 \mathrm{H}), 0.93(\mathrm{~s}, 10 \mathrm{H}), 0.86(\mathrm{~d}, J=6.9$ $\mathrm{Hz}, 1 \mathrm{H}), 0.14(\mathrm{~s}, 3 \mathrm{H}), 0.06(\mathrm{~s}, 3 \mathrm{H})$.

${ }^{13}$ C-NMR: (126 MHz, Chloroform-d) $\delta$ 152.9, 120.3, 61.2, 60.0, 53.7, 52.1, 40.7, 32.8, 31.1, 30.8, 30.0, $28.2,26.1,25.5,23.8,22.0,20.8,20.4,20.2,18.3,15.7,14.3,-3.1,-4.9$.

IR (thin film, cm ${ }^{-1}$ ): 2954, 2928, 2857, 1718, 1698, 1649, 1463, 1422, 1377, 1304, 1254, 1180, 1146, $1089,1054,1034,1005,989,938,904,835,776,729,670,588$

HRMS (ESI): $m / z$ : exact mass calculated for $\mathrm{C}_{29} \mathrm{H}_{51} \mathrm{O}_{4} \mathrm{~S}_{2} \mathrm{Si}[\mathrm{M}+\mathrm{H}]^{+}, 555.2993$; found: 555.2991.

$[\mathrm{a}]_{\mathrm{D}}^{25^{\circ}}=273.5\left(\mathrm{c}=0.4, \mathrm{CHCl}_{3}\right)$ 


\section{Supporting Information}
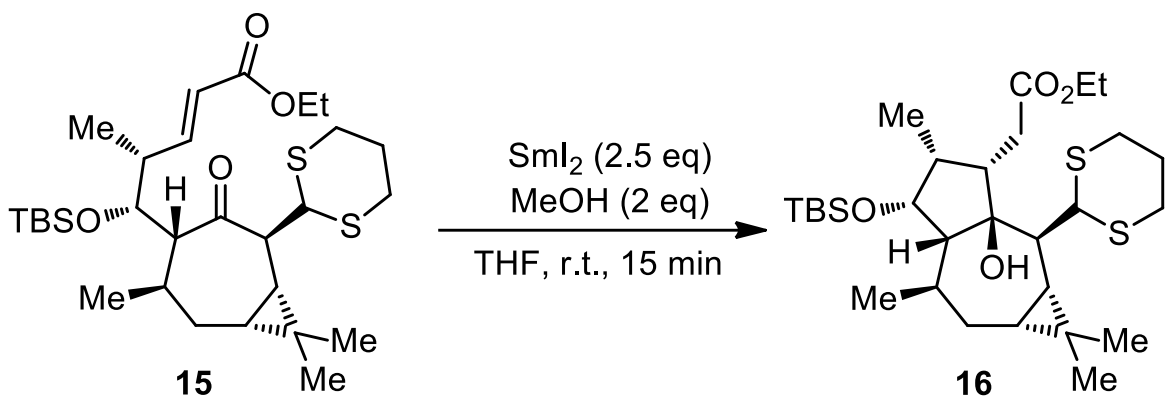

To a solution of $15(8.0 \mathrm{mg}, 0.014 \mathrm{mmol}, 1.0 \mathrm{eq})$ in degassed THF ( $2 \mathrm{~mL})$ under argon was added $\mathrm{MeOH}$ ( $2.3 \mu \mathrm{L}, 0.058 \mathrm{mmol}, 2.5$ eq). While stirring a $0.1 \mathrm{M} \mathrm{Sml}_{2}$ solution in THF (0.36 mL, $0.036 \mathrm{mmol}, 2.5$ eq) was added until the blue color persisted. After stirring at room temperature for 25 minutes the reaction was quenched with sat. aq. $\mathrm{NaHCO}_{3}(3 \mathrm{~mL})$ and sat. aq. $\mathrm{Na}_{2} \mathrm{~S}_{2} \mathrm{O}_{3}(3 \mathrm{~mL})$, extracted with diethyl ether $\left(3 \times 10 \mathrm{~mL}\right.$ ), dried over $\mathrm{Na}_{2} \mathrm{SO}_{4}$ and concentrated in vacuo. After purification via flash column chromatography (hexane- $\left.\mathrm{Et}_{2} \mathrm{O}, 75: 25\right)$ the purified product $16(6.3 \mathrm{mg}, 0.011 \mathrm{mmol}, 78 \%)$ was obtained as a pale-yellow oil.

${ }^{1} \mathrm{H}-\mathrm{NMR}(500 \mathrm{MHz}$, Chloroform-d) $\delta 4.46(\mathrm{~d}, J=2.3 \mathrm{~Hz}, 1 \mathrm{H}), 4.39(\mathrm{t}, J=6.5 \mathrm{~Hz}, 1 \mathrm{H}), 4.19-4.09(\mathrm{~m}, 2 \mathrm{H})$, $3.06-2.99(\mathrm{~m}, 2 \mathrm{H}), 2.82-2.71(\mathrm{~m}, 4 \mathrm{H}), 2.55(\mathrm{ddd}, J=9.5,6.4,3.2 \mathrm{~Hz}, 1 \mathrm{H}), 2.37-2.29(\mathrm{~m}, 1 \mathrm{H}), 2.16$ (ddt, $J=8.8,6.4,3.2 \mathrm{~Hz}, 1 \mathrm{H}), 2.11-2.06(\mathrm{~m}, 1 \mathrm{H}), 1.98-1.85(\mathrm{~m}, 3 \mathrm{H}), 1.51-1.47(\mathrm{~m}, 1 \mathrm{H}), 1.41-1.35$ $(\mathrm{m}, 1 \mathrm{H}), 1.26(\mathrm{~d}, J=6.7 \mathrm{~Hz}, 3 \mathrm{H}), 1.12(\mathrm{~d}, J=2.4 \mathrm{~Hz}, 6 \mathrm{H}), 1.02(\mathrm{~d}, J=6.6 \mathrm{~Hz}, 3 \mathrm{H}), 0.96-0.90(\mathrm{~m}, 13 \mathrm{H})$, $0.85(\mathrm{dd}, J=5.9,3.2 \mathrm{~Hz}, 1 \mathrm{H}), 0.07(\mathrm{~s}, 3 \mathrm{H}), 0.05(\mathrm{~s}, 3 \mathrm{H})$.

${ }^{13}$ C-NMR: (126 MHz, Chloroform-d) $\delta 90.2,61.9,60.6,52.5,50.6,43.8,38.1,33.0,32.2,32.1,29.9,29.2$, 29.1, 28.7, 26.6, 26.4, 25.1, 24.9, 20.9, 19.0, 17.4, 14.3, 12.3, -3.0, -3.9.

IR (thin film, $\mathrm{cm}^{-1}$ ): 2930, 2926, 2820, 1756, 1750. 1733, 1490, 1375, 1249, 830.

HRMS (ESI): $m / z$ : exact mass calculated for $\mathrm{C}_{29} \mathrm{H}_{52} \mathrm{NaO}_{4} \mathrm{~S}_{2} \mathrm{Si}[\mathrm{M}+\mathrm{Na}]^{+}, 579.2968$; found: 579.2964 . $[\mathrm{a}]_{\mathrm{D}}{ }^{25^{\circ}}=-0.4\left(\mathrm{c}=0.2, \mathrm{CHCl}_{3}\right)$. 


\section{Supporting Information}
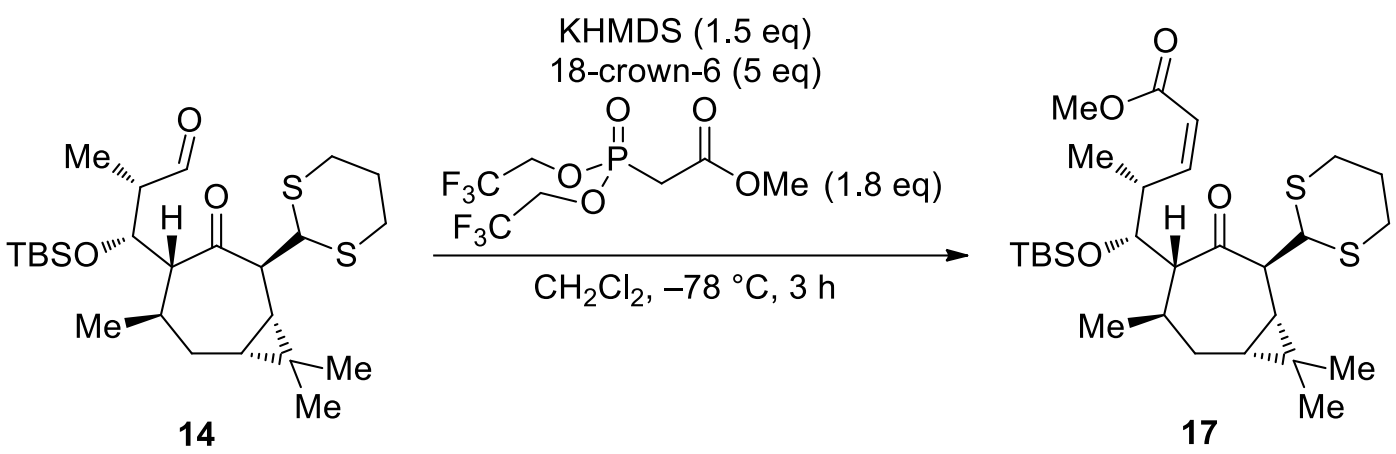

To a well stirred solution of 18 -crown- $6(1.36 \mathrm{~g}, 5.16 \mathrm{mmol}, 5.00 \mathrm{eq})$ in THF $(16 \mathrm{~mL})$ at $-78^{\circ} \mathrm{C}$ was added 0.5 M solution of KHDMS in PhMe $(3.09 \mathrm{~mL}, 1.55 \mathrm{mmol}, 1.5 \mathrm{eq})$ and the reaction was stirred for 20 minutes. After addition of 14 ( $0.500 \mathrm{~g}, 1.03 \mathrm{mmol}, 1.0 \mathrm{eq})$ and stirring for 3 hour the reaction was quenched with sat. aq. $\mathrm{NH}_{4} \mathrm{Cl}(10 \mathrm{~mL})$, extracted with diethyl ether $(3 \times 50 \mathrm{~mL})$, dried over $\mathrm{Na}_{2} \mathrm{SO}_{4}$ and concentrated in vacuo. Purification by flash column chromatography (hexane- $\mathrm{Et}_{2} \mathrm{O}, 97: 3$ ) yielded the product 17 (435 mg, $0.804 \mathrm{mmol}, 78 \%$ ) as a pure, pale yellow, amorphous solid. No E-isomer was observed.

${ }^{1} \mathrm{H}-\mathrm{NMR}\left(400 \mathrm{MHz}, \mathrm{CDCl}_{3}\right) \delta 6.71(\mathrm{dd}, J=11.8,10.1 \mathrm{~Hz}, 1 \mathrm{H}), 5.70(\mathrm{~d}, J=11.8 \mathrm{~Hz}, 1 \mathrm{H}), 4.61(\mathrm{~d}$, $J=5.2 \mathrm{~Hz}, 1 \mathrm{H}), 4.24-4.07(\mathrm{~m}, 1 \mathrm{H}), 3.98(\mathrm{~s}, 1 \mathrm{H}), 3.70(\mathrm{~s}, 3 \mathrm{H}), 3.10(\mathrm{~d}, J=8.3 \mathrm{~Hz}, 1 \mathrm{H}), 3.05-2.88(\mathrm{~m}$, $2 \mathrm{H}), 2.86-2.76(\mathrm{~m}, 2 \mathrm{H}), 2.42(\mathrm{dd}, J=8.4,5.3 \mathrm{~Hz}, 1 \mathrm{H}), 2.23-2.10(\mathrm{~m}, 1 \mathrm{H}), 2.13-2.02(\mathrm{~m}, 1 \mathrm{H}), 1.99-$ $1.78(\mathrm{~m}, 1 \mathrm{H}), 1.55-1.47(\mathrm{~m}, 1 \mathrm{H}), 1.17-1.08(\mathrm{~m}, 9 \mathrm{H}), 0.97(\mathrm{~d}, J=2.2 \mathrm{~Hz}, 2 \mathrm{H}), 0.95(\mathrm{~s}, 3 \mathrm{H}), 0.92(\mathrm{~s}$, 9H), $0.87(\mathrm{~d}, J=7.4 \mathrm{~Hz}, 1 \mathrm{H}), 0.17(\mathrm{~s}, 3 \mathrm{H}), 0.06(\mathrm{~s}, 3 \mathrm{H})$.

${ }^{13}$ C-NMR: $\left(101 \mathrm{MHz}, \mathrm{CDCl}_{3}\right) \delta$ 213.9, 166.8, 153.2, 116.9, 75.9, 61.6, 53.8, 52.5, 51.1, 36.5, 32.0, 31.0, $30.6,30.1,28.2,26.1,25.5,23.3,21.9,21.2,20.8,20.3,18.2,15.6,-3.3,-5.1$.

IR (thin film, $\mathrm{cm}^{-1}$ ): 2953, 2929, 2858, 1723, 1697, 1463, 1435, 1276, 1253, 1193, 1176, 1114, 1088, $1053,1034,1023,1006,902,836,775$.

HRMS (ESI): $m / z$ : exact mass calculated for $\mathrm{C}_{28} \mathrm{H}_{48} \mathrm{NaO}_{4} \mathrm{~S}_{2} \mathrm{Si}[\mathrm{M}+\mathrm{Na}]^{+}, 563.2655$; found: 563.2645 .

$[a]_{D}^{25^{\circ}}=69.5\left(\mathrm{c}=1.0, \mathrm{CHCl}_{3}\right)$ 


\section{Supporting Information}

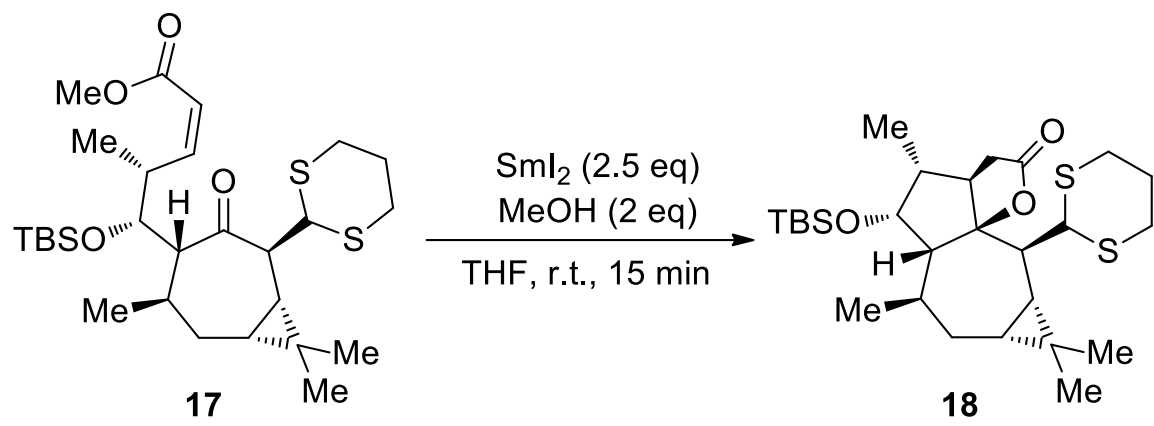

To a solution of 17 (412 mg, $0.609 \mathrm{mmol}, 1.0 \mathrm{eq})$ in degassed THF (20 mL) under argon was added $\mathrm{MeOH}(99.0 \mu \mathrm{L}, 2.44 \mathrm{mmol}, 4.0 \mathrm{eq})$. While stirring a $0.1 \mathrm{M} \mathrm{Sml}_{2}$ solution in THF (14.8 mL, $1.48 \mathrm{mmol}$, 2.5 eq) was added until the blue color persisted. After stirring at room temperature for 15 minutes the reaction was quenched with sat. aq. $\mathrm{NaHCO}_{3}(15 \mathrm{~mL})$ and sat. aq. $\mathrm{Na}_{2} \mathrm{~S}_{2} \mathrm{O}_{3}(15 \mathrm{~mL})$, extracted with diethyl ether $\left(3 \times 50 \mathrm{~mL}\right.$ ), dried over $\mathrm{Na}_{2} \mathrm{SO}_{4}$ and concentrated in vacuo. After purification via flash column chromatography (hexane-Et $\left.{ }_{2} \mathrm{O}, 90: 10\right)$ the purified product 18 ( $277 \mathrm{mg}, 0.542 \mathrm{mmol}, 89 \%$ ) was obtained as a pale-yellow oil.

${ }^{1} \mathrm{H}-\mathrm{NMR}\left(400 \mathrm{MHz}, \mathrm{CDCl}_{3}\right) \delta 4.40(\mathrm{~d}, J=3.1 \mathrm{~Hz}, 1 \mathrm{H}), 4.03(\mathrm{t}, J=2.6 \mathrm{~Hz}, 1 \mathrm{H}), 3.25-3.12(\mathrm{~m}, 1 \mathrm{H}), 3.01-$ $2.79(\mathrm{~m}, 4 \mathrm{H}), 2.65(\mathrm{td}, J=10.4,2.5 \mathrm{~Hz}, 1 \mathrm{H}), 2.32(\mathrm{dd}, J=10.1,3.1 \mathrm{~Hz}, 1 \mathrm{H}), 2.29-2.17(\mathrm{~m}, 1 \mathrm{H}), 2.06(\mathrm{dtt}$, $J=13.5,4.0,2.5 \mathrm{~Hz}, 1 \mathrm{H}), 1.94-1.53(\mathrm{~m}, 6 \mathrm{H}), 1.08(\mathrm{~s}, 7 \mathrm{H}), 1.05(\mathrm{~d}, J=6.7 \mathrm{~Hz}, 3 \mathrm{H}), 0.92(\mathrm{~s}, 9 \mathrm{H}), 0.89$ (d, $J=6.1 \mathrm{~Hz}, 3 \mathrm{H}$ ), 0.80 (ddd, $J=10.0,6.9,1.9 \mathrm{~Hz}, 1 \mathrm{H}), 0.06(\mathrm{~d}, J=9.8 \mathrm{~Hz}, 6 \mathrm{H}$ ).

${ }^{13}$ C-NMR: $\left(101 \mathrm{MHz} \mathrm{CDCl}_{3}\right) \delta$ 177.7, 98.4, 78.0, 66.1, 53.7, 46.4, 46.1, 45.1, 37.3, 32.2, 32.0, 31.2, 29.5, $27.5,26.4,26.1,25.8,23.1,22.9,19.0,18.4,16.2,14.1,-3.4,-4.3$.

IR (thin film, cm ${ }^{-1}$ ): 2954, 2929 2892, 2858, 1767, 1461, 1422, 1413, 1376, 1361, 1333, 1303, 1276, $1254,1208,1194,1152,1138,1099,1081,1022,987,963,941,914,859,835,802,773,756,710,666$, $646,607,547,465$.

HRMS (ESI): $m / z$ : exact mass calculated for $\mathrm{C}_{27} \mathrm{H}_{46} \mathrm{NaO}_{3} \mathrm{~S}_{2} \mathrm{Si}$ [M+Na] ${ }^{+}, 533.255$; found: 533.2548 .

$[a]_{D}^{25^{\circ}}=65.9\left(\mathrm{c}=1.0, \mathrm{CHCl}_{3}\right)$ 


\section{Supporting Information}
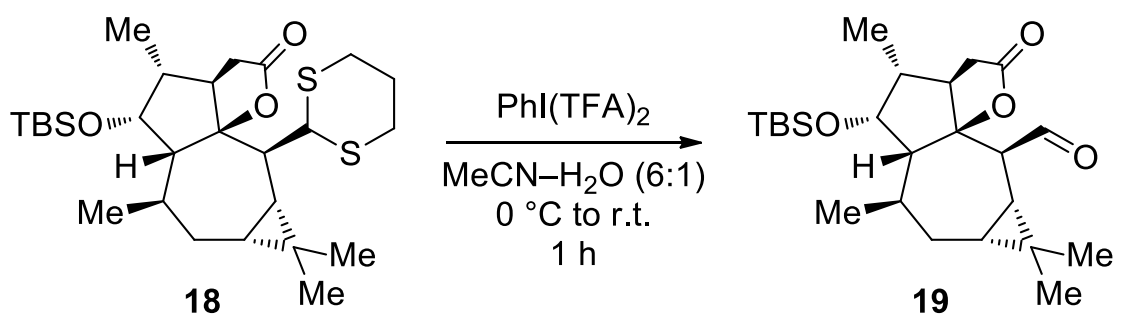

To a solution of lactone 18 (211 mg, $0.413 \mathrm{mmol}, 1.0 \mathrm{eq})$ in $\mathrm{MeCN}-\mathrm{H}_{2} \mathrm{O}(6: 1,7 \mathrm{~mL})$ was added PIFA (444 mg, $1.03 \mathrm{mmol}, 2.5 \mathrm{eq}$ ) at $0^{\circ} \mathrm{C}$. The mixture was warmed to room temperature and stirred for $1 \mathrm{~h}$. After complete consumption of starting material, the mixture was transferred to a separation funnel and extracted with $\mathrm{Et}_{2} \mathrm{O}(3 \times 30 \mathrm{~mL})$. The organic phases were combined, dried over $\mathrm{Na}_{2} \mathrm{SO}_{4}$, and concentrated under reduced pressure. The crude product was purified by flash column chromatography (hexane-Et ${ }_{2} \mathrm{O}, 80: 20$ ) to give 19 as colorless oil (165 mg, $0.392 \mathrm{mmol}, 95 \%$ ), which solidified in the freezer.

${ }^{1} \mathrm{H}-\mathrm{NMR}\left(400 \mathrm{MHz}, \mathrm{CDCl}_{3}\right) \delta 9.55(\mathrm{~d}, J=2.4 \mathrm{~Hz}, 1 \mathrm{H}), 4.00(\mathrm{t}, J=3.0 \mathrm{~Hz}, 1 \mathrm{H}), 3.01(\mathrm{dd}, J=18.4,10.4 \mathrm{~Hz}$, 1H), 2.79 (dd, $J=11.3,2.4 \mathrm{~Hz}, 1 \mathrm{H}$ ), 2.36 (td, $J=10.6,2.8 \mathrm{~Hz}, 1 \mathrm{H}$ ), 2.24 (dd, $J=18.6,2.8 \mathrm{~Hz}, 1 \mathrm{H}$ ), 2.07 (ddd, $J=6.8,4.2,2.5 \mathrm{~Hz}, 1 \mathrm{H}$ ), $1.83(\mathrm{dd}, J=4.7,3.0 \mathrm{~Hz}, 1 \mathrm{H}), 1.82-1.77(\mathrm{~m}, 2 \mathrm{H}), 1.68$ (dqd, $J=9.8,6.7$, $2.9 \mathrm{~Hz}, 1 \mathrm{H}), 1.14(\mathrm{~s}, 3 \mathrm{H}), 1.10(\mathrm{~d}, J=7.0 \mathrm{~Hz}, 3 \mathrm{H}), 1.07(\mathrm{~s}, 3 \mathrm{H}), 0.99-0.94(\mathrm{~m}, 4 \mathrm{H}), 0.92(\mathrm{~s}, 9 \mathrm{H}), 0.84(\mathrm{dd}$, $J=11.3,9.4 \mathrm{~Hz}, 1 \mathrm{H}), 0.08-0.07(\mathrm{~m}, 6 \mathrm{H})$.

${ }^{13} \mathrm{C}-\mathrm{NMR}\left(101 \mathrm{MHz}, \mathrm{CDCl}_{3}\right) \delta$ 200.3, 177.6, 95.9, 80.7, 63.2, 55.4, 48.8, 46.4, 35.5, 30.3, 29.1, 29.0, 26.4, 23.6, 22.9, 22.4, 19.9, 18.3, 15.7, 13.9, -3.4, -4.3.

IR (thin film, cm ${ }^{-1}$ ): 2927, 1776, 1728, 1462, 1253, 1029, 836, 775.

HRMS (ESI): $m / z$ : exact mass calculated for $\mathrm{C}_{24} \mathrm{H}_{40} \mathrm{NaO}_{4} \mathrm{Si}[\mathrm{M}+\mathrm{Na}]^{+}, 443.2588$; found: 443.2589 .

$[a]_{D}{ }^{25^{\circ}}=-1.3\left(\mathrm{c}=0.3, \mathrm{CHCl}_{3}\right)$. 


\section{Supporting Information}

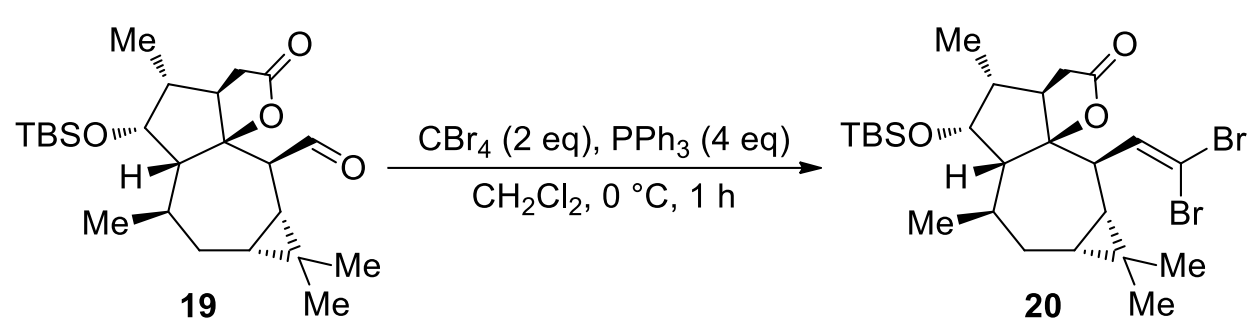

To a well stirred solution of tetrabromomethane $(237 \mathrm{mg}, 0.713 \mathrm{mmol}, 2.0 \mathrm{eq})$ in $\mathrm{CH}_{2} \mathrm{Cl}_{2}(3.5 \mathrm{~mL}$ ) at $0{ }^{\circ} \mathrm{C}$ was added triphenylphosphine $(374 \mathrm{mg}, 1.43 \mathrm{mmol}, 4.0 \mathrm{eq})$ and the solution was stirred for $30 \mathrm{~min}$ at $0{ }^{\circ} \mathrm{C}$. After addition of $19(150 \mathrm{mg}, 0.357 \mathrm{mmol}, 1.0 \mathrm{eq})$ in $\mathrm{CH}_{2} \mathrm{Cl}_{2}(0.5 \mathrm{~mL})$ and stirring at $0{ }^{\circ} \mathrm{C}$ for $1 \mathrm{~h}$, the reaction was quenched with sat. aq. $\mathrm{NH}_{4} \mathrm{Cl}(10 \mathrm{~mL})$, extracted with $\mathrm{CH}_{2} \mathrm{Cl}_{2}(3 \times 20 \mathrm{~mL})$, dried over $\mathrm{Na}_{2} \mathrm{SO}_{4}$ and concentrated in vacuo. Flash column chromatography (hexane-Et ${ }_{2} \mathrm{O}$ 90:10) yielded the pure product 20 (148 $\mathrm{mg}, 0.257 \mathrm{mmol}, 72 \%$ ) as a colorless oil.

${ }^{1} \mathrm{H}-\mathrm{NMR}(400 \mathrm{MHz}$, Chloroform-d) $\delta 6.27(\mathrm{~d}, J=10.2 \mathrm{~Hz}, 1 \mathrm{H}), 4.00(\mathrm{t}, J=3.3 \mathrm{~Hz}, 1 \mathrm{H}), 2.73-2.60(\mathrm{~m}$, $2 \mathrm{H}), 2.34-2.16(\mathrm{~m}, 3 \mathrm{H}), 1.93(\mathrm{t}, J=3.1 \mathrm{~Hz}, 1 \mathrm{H}), 1.79-1.62(\mathrm{~m}, 3 \mathrm{H}), 1.15(\mathrm{~d}, J=7.2 \mathrm{~Hz}, 3 \mathrm{H}), 1.10(\mathrm{~s}$, $3 \mathrm{H}), 1.03(\mathrm{~s}, 3 \mathrm{H}), 0.99(\mathrm{~d}, J=6.7 \mathrm{~Hz}, 3 \mathrm{H}), 0.93(\mathrm{~s}, 9 \mathrm{H}), 0.80$ (dd, $J=9.6,5.7 \mathrm{~Hz}, 1 \mathrm{H}), 0.73(\mathrm{t}, J=9.6 \mathrm{~Hz}$, $1 \mathrm{H}), 0.08(\mathrm{~s}, 6 \mathrm{H})$.

${ }^{13}$ C-NMR (101 MHz, Chloroform-d) $\delta$ 138.6, 99.7, 81.9, 62.5, 48.8, 48.8, 45.8, 35.0, 31.4, 29.2, 28.3, 26.7, 26.5, 23.7, 22.6, 20.1, 18.5, 16.3, 14.1, -3.6, -3.9.

IR: $2955,2929,2859,1775,1462,1377,1257,1193,1154,1118,1072,1024,1004,959,940,872,836$, $800,774,751$.

HRMS (ESI) $m / z$ : exact mass calculated for $\mathrm{C}_{25} \mathrm{H}_{40} \mathrm{Br}_{2} \mathrm{NaO}_{3} \mathrm{Si}[\mathrm{M}+\mathrm{Na}]^{+}$, 597.1006; found: 597.1011. $[a]_{D}^{25^{\circ}}=-0.4\left(c=1.0, \mathrm{CHCl}_{3}\right)$. 


\section{Supporting Information}
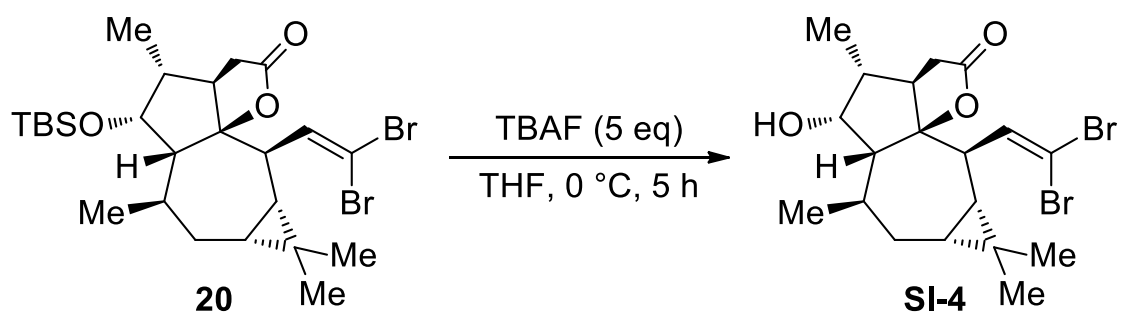

To a well stirred solution of $20(0.050 \mathrm{~g}, 0.087 \mathrm{mmol}, 1.0 \mathrm{eq})$ in THF $(1.7 \mathrm{~mL})$ at $0{ }^{\circ} \mathrm{C}$ was added a $1 \mathrm{M}$ solution of TBAF in THF $(0.434 \mathrm{~mL}, 0.434 \mathrm{mmol}, 5.0 \mathrm{eq})$ and the reaction was stirred at $0{ }^{\circ} \mathrm{C}$ for $5 \mathrm{~h}$. After quenching the reaction with sat. aq. $\mathrm{NaHCO}_{3}$ and extracting with diethyl ether $(3 \times 20 \mathrm{~mL})$, the organic phases were dried over $\mathrm{Na}_{2} \mathrm{SO}_{4}$, concentrated in vacuo, and purified by flash column chromatography (hexane-Et ${ }_{2} \mathrm{O} 80: 20$ to $50: 50$ ), yielding the desired product SI-4 (36 mg, 0.078 mmol, $90 \%)$ colorless oil.

${ }^{1} \mathrm{H}-\mathrm{NMR}(400 \mathrm{MHz}$, Chloroform-d) $\delta 6.29(\mathrm{~d}, J=10.2 \mathrm{~Hz}, 1 \mathrm{H}), 4.03(\mathrm{q}, J=3.3 \mathrm{~Hz}, 1 \mathrm{H}), 2.79$ (t, $J=9.9 \mathrm{~Hz}$, $1 \mathrm{H}), 2.70(\mathrm{dd}, J=18.2,9.3 \mathrm{~Hz}, 1 \mathrm{H}), 2.37-2.25(\mathrm{~m}, 2 \mathrm{H}), 2.05-1.91(\mathrm{~m}, 2 \mathrm{H}), 1.88-1.74(\mathrm{~m}, 2 \mathrm{H}), 1.66$ (ddd, $J=10.4,6.8,3.2 \mathrm{~Hz}, 1 \mathrm{H}$ ), $1.12(\mathrm{~s}, 3 \mathrm{H}), 1.07$ (d, J = 5.3 Hz, 3H), 1.05 (d, J = 5.2 Hz, 3H), $1.03(\mathrm{~s}, 3 \mathrm{H}$ ), 0.79 (ddd, $J=9.6,7.3,4.2 \mathrm{~Hz}, 1 \mathrm{H}), 0.69(\mathrm{t}, J=9.6 \mathrm{~Hz}, 1 \mathrm{H}$ ).

${ }^{13} \mathrm{C}-N M R(101 \mathrm{MHz}$, Chloroform-d) $\delta 177.3,138.8,99.1,90.4,78.9,62.4,48.2,47.2,44.2,34.7,31.5$, $30.1,29.4,28.2,23.5,22.2,19.2,16.1,12.6$.

IR: 3483, 2954, 2926, 1761, 1459, 1377, 1198, 1155, 1136, 1089, 1066, 1021, 1002, 961, 921, 805, 777, $747,695,540$.

HRMS (ESI) $\mathrm{m} / \mathrm{z}$ : exact mass calculated for $\mathrm{C}_{19} \mathrm{H}_{26} \mathrm{Br}_{2} \mathrm{NaO}_{3}$ [M+Na] ${ }^{+}, 483.0141$; found: 483.0132 .

$[a]_{D}{ }^{25^{\circ}}=-29.2\left(\mathrm{c}=0.9, \mathrm{CHCl}_{3}\right)$ 


\section{Supporting Information}
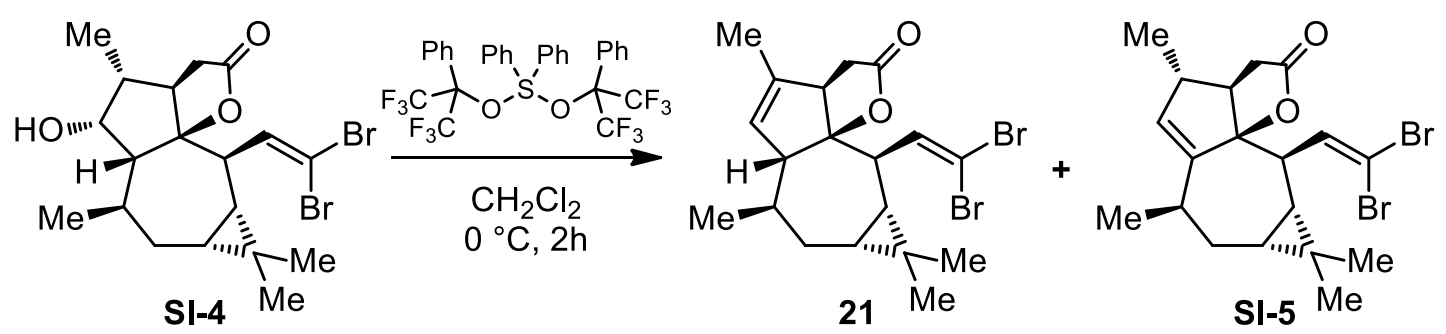

To a solution of alcohol SI-4 (0.030 g, 0.065 mmol, 1.0 eq) in $\mathrm{CH}_{2} \mathrm{Cl}_{2}$ was added Martin's Sulfurane (66 mg, $0.097 \mathrm{mmol}, 1.5 \mathrm{eq}$ ) at $0{ }^{\circ} \mathrm{C}$ and the solution was stirred at the same temperature for $2 \mathrm{~h}$. Sat. aq. $\mathrm{NaHCO}_{3}(5 \mathrm{~mL})$ was added to the mixture to quench the reaction. The mixture was transferred to a separation funnel and extracted with $\mathrm{Et}_{2} \mathrm{O}(3 \times 20 \mathrm{~mL})$. The combined organic phases were dried over $\mathrm{Na}_{2} \mathrm{SO}_{4}$, filtered and concentrated under reduced pressure, to give crude 21. Purification by flash column chromatography (hexane- $\left.\mathrm{Et}_{2} \mathrm{O}, 90: 10\right)$ gave 21 (12 mg, $\left.0.027 \mathrm{mmol}, 42 \%\right)$ and SI-5 (8.5 mg, $0.019 \mathrm{mmol}, 30 \%$ ) as colorless oils that solidified in the freezer.

Analytical data for $\mathbf{2 1 :}$

${ }^{1} \mathrm{H}-\mathrm{NMR}(400 \mathrm{MHz}$, Chloroform-d) $\delta 6.34(\mathrm{~d}, J=10.3 \mathrm{~Hz}, 1 \mathrm{H}$ ), 5.50 (d, $J=2.3 \mathrm{~Hz}, 1 \mathrm{H}$ ), 2.91 (ddd, $J=7.2$, 4.9, 2.2 Hz, 1H), 2.82 (dd, J = 18.4, 10.4 Hz, 1H), $2.45-2.27(\mathrm{~m}, 4 \mathrm{H}), 1.95$ (ddd, J = 14.5, 6.0, $4.8 \mathrm{~Hz}, 1 \mathrm{H}$ ), $1.37-1.28(\mathrm{~m}, 1 \mathrm{H}), 1.25(\mathrm{~d}, J=7.4 \mathrm{~Hz}, 3 \mathrm{H}), 1.11(\mathrm{~d}, J=7.0 \mathrm{~Hz}, 3 \mathrm{H}), 1.08(\mathrm{~s}, 3 \mathrm{H}), 1.08(\mathrm{~s}, 3 \mathrm{H}), 0.92$ (ddd, $J=11.6,9.3,6.0 \mathrm{~Hz}, 1 \mathrm{H}), 0.83(\mathrm{t}, J=9.6 \mathrm{~Hz}, 1 \mathrm{H})$.

${ }^{13}$ C-NMR: (101 MHz, Chloroform-d) $\delta$ 177.1, 148.6, 138.0, 136.7, 103.8, 89.9, 50.0, 49.3, 45.4, 36.6, $36.2,29.5,29.0,27.7,24.1,21.2,20.0,18.9,16.4$.

IR (thin film, $\mathrm{cm}^{-1}$ ): 2927, 1775, 1459, 1377, 1198, 1146, 1007, 960, 801.

HRMS (ESI): $m / z$ : exact mass calculated for $\mathrm{C}_{19} \mathrm{H}_{24} \mathrm{Br}_{2} \mathrm{NaO}_{2}[\mathrm{M}+\mathrm{Na}]^{+}, 465.0035$; found: 465.0028.

$[a]_{D}^{25^{\circ}}=-71.6\left(\mathrm{c}=0.5, \mathrm{CHCl}_{3}\right)$

Analytical data for SI-5:

${ }^{1} \mathrm{H}-\mathrm{NMR}(400 \mathrm{MHz}$, Chloroform-d) $\delta 6.41(\mathrm{~d}, J=10.0 \mathrm{~Hz}, 1 \mathrm{H}), 5.41(\mathrm{dt}, J=2.8,1.6 \mathrm{~Hz}, 1 \mathrm{H}), 3.03(\mathrm{dq}, J=$ 9.3, $1.5 \mathrm{~Hz}, 1 \mathrm{H}), 2.73(\mathrm{dd}, J=10.0,2.2 \mathrm{~Hz}, 1 \mathrm{H}), 2.70-2.58(\mathrm{~m}, 2 \mathrm{H}), 2.40(\mathrm{dt}, J=18.2,0.9 \mathrm{~Hz}, 1 \mathrm{H}), 1.83$ (ddd, $J=15.3,6.0,3.6 \mathrm{~Hz}, 1 \mathrm{H}$ ), $1.75-1.68(\mathrm{~m}, 1 \mathrm{H}$ ), $1.66(\mathrm{p}, J=1.6 \mathrm{~Hz}, 3 \mathrm{H}$ ), 1.39 (ddd, $J=16.7,10.4$, $6.1 \mathrm{~Hz}, 1 \mathrm{H}$ ), $1.03(\mathrm{~s}, 6 \mathrm{H}), 0.99$ (dd, $J=10.5,9.4 \mathrm{~Hz}, 1 \mathrm{H}$ ), 0.90 (d, J = 6.5 Hz, 3H), 0.75 (td, $J=9.5,3.5 \mathrm{~Hz}$, $1 \mathrm{H})$.

${ }^{13}$ C-NMR: (101 MHz, Chloroform-d) $\delta$ 176.5, 138.3, 137.3, 128.4, 100.9, 58.0, 51.0, 45.9, 33.9, 33.3, $32.3,29.1,28.6,21.7,21.5,18.9,16.4,14.4$.

IR (thin film, cm $\mathrm{cm}^{-1}$ ): 1145, 1011, 970, 937, 918, 855, 820, 799, 781, 737, 540, 459,

HRMS (ESI): $\mathrm{m} / z$ : exact mass calculated for $\mathrm{C}_{19} \mathrm{H}_{25} \mathrm{Br}_{2} \mathrm{O}_{2}[\mathrm{M}+\mathrm{H}]^{+}, 443.0216$; found: 443.0218

$[a]_{D}^{25^{\circ}}=-5.5\left(c=0.3, \mathrm{CHCl}_{3}\right)$ 


\section{Supporting Information}

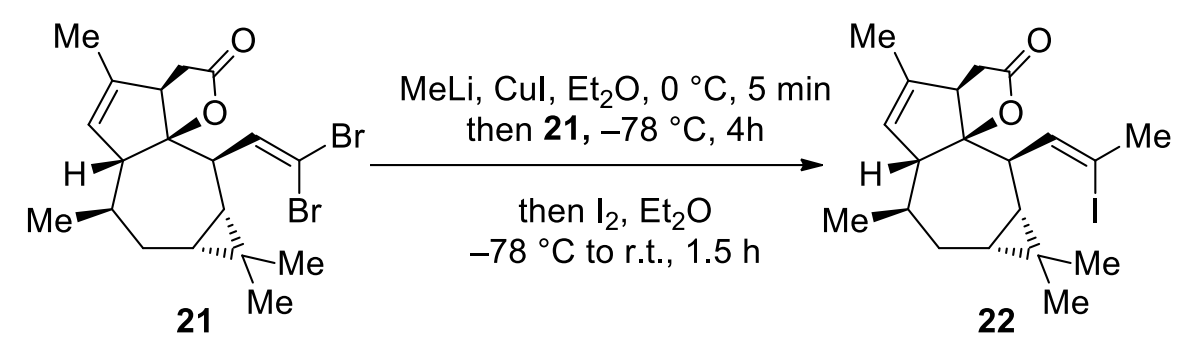

MeLi (1.6M in Et ${ }_{2} \mathrm{O}, 0.093 \mathrm{~mL}, 0.15 \mathrm{mmol}, 6.0 \mathrm{eq}$ ) was added to a suspension of Cul (14 mg, $0.074 \mathrm{mmol}, 3.0 \mathrm{eq})$ in $\mathrm{Et}_{2} \mathrm{O}(0.5 \mathrm{~mL})$ at $0{ }^{\circ} \mathrm{C}$ and stirred for $5 \mathrm{~min}$. The now clear solution as cooled to $-78{ }^{\circ} \mathrm{C}$ and a solution of 21 (11 mg, $\left.0.025 \mathrm{mmol}, 1.0 \mathrm{eq}\right)$ in $\mathrm{Et}_{2} \mathrm{O}(0.2 \mathrm{~mL})$ was added dropwise. The temperature was maintained, and the solution was stirred for $5 \mathrm{~h}$. lodine ( $38 \mathrm{mg}, 0.15 \mathrm{mmol}, 6.0 \mathrm{eq}$ ) in $\mathrm{Et}_{2} \mathrm{O}(3 \mathrm{~mL})$ was added dropwise and the mixture was stirred for $20 \mathrm{~min}$ at $-78^{\circ} \mathrm{C}$ and $1 \mathrm{~h}$ at room temperature. Sat aq. $\mathrm{Na}_{2} \mathrm{SO}_{3}(10 \mathrm{~mL})$ was added, and the mixture was transferred to a separation funnel with distilled water $(10 \mathrm{~mL})$. The mixture was extracted with $\mathrm{Et}_{2} \mathrm{O}(3 \times 15 \mathrm{~mL})$, the organic phases were combined, dried over $\mathrm{Na}_{2} \mathrm{SO}_{4}$, filtered and concentrated under reduced pressure. The crude product was purified by flash column chromatography (hexane-Et ${ }_{2} \mathrm{O}, 90: 10$ ) to give $\mathbf{2 2}$ (contaminated by small amounts of the bromomethyl and dimethyl species) as a colorless oil $(7.8 \mathrm{mg}, 0.018 \mathrm{mmol}$, 74\%).

${ }^{1} \mathrm{H}-\mathrm{NMR}(400 \mathrm{MHz}$, Chloroform-d) $\delta 5.48(\mathrm{~d}, J=2.1 \mathrm{~Hz}, 1 \mathrm{H}$ ), 5.37 (dd, $J=9.6,1.5 \mathrm{~Hz}, 1 \mathrm{H}), 2.91$ (dtd, $J=$ 9.7, 4.9, 2.3 Hz, 1H), $2.77-2.69(\mathrm{~m}, 1 \mathrm{H}), 2.56(\mathrm{~d}, J=1.5 \mathrm{~Hz}, 3 \mathrm{H}), 2.38-2.30(\mathrm{~m}, 4 \mathrm{H}), 1.95-1.89(\mathrm{~m}$, $1 \mathrm{H}), 1.39-1.33(\mathrm{~m}, 1 \mathrm{H}), 1.25(\mathrm{~s}, 3 \mathrm{H}), 1.15(\mathrm{~s}, 3 \mathrm{H}), 1.12(\mathrm{~s}, 3 \mathrm{H}), 1.05(\mathrm{~s}, 3 \mathrm{H}), 0.89-0.82(\mathrm{~m}, 2 \mathrm{H})$.

${ }^{13}$ C-NMR: (101 MHz, Chloroform-d) $\delta$ 177.7, 148.9, 136.8, 135.3, 105.0, 101.9, 52.6, 48.9, 45.7, 36.7, $36.3,34.4,29.3,29.2,28.0,23.8,21.3,20.0,19.2,17.4$.

IR (thin film, cm ${ }^{-1}$ ): 2915, 2868, 1775, 1444, 1417, 1376, 1299, 1247, 1218, 1187, 1147, 1066, 1014, $979,967,937,915,865,787,753,653,574,539,508,470,423,410$.

HRMS (ESI): $m / z$ : exact mass calculated for $\mathrm{C}_{20} \mathrm{H}_{27} \mathrm{INaO}_{2}$ [M+Na] ${ }^{+}$, 449.0948; found: 449.0943 .

[]$_{\mathrm{D}}{ }^{25^{\circ}}=-53.7\left(\mathrm{c}=1.0, \mathrm{CHCl}_{3}\right)$ 


\section{Supporting Information}

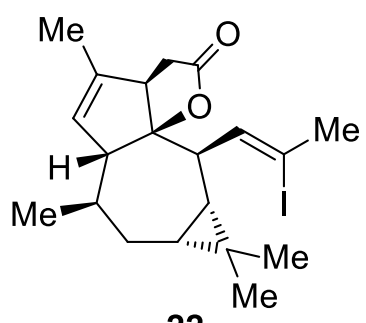

22
KHMDS, THF, $-78^{\circ} \mathrm{C}, 15 \mathrm{~min}$<smiles>ON(OP(O)c1ccccc1)c1ccccc1</smiles>

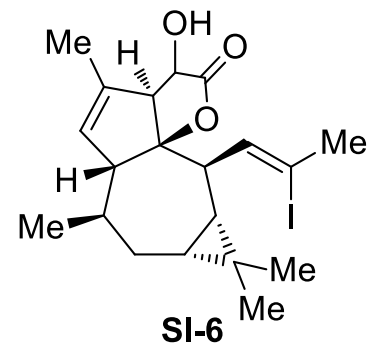

SI-6

$-78{ }^{\circ} \mathrm{C}$ to r.t., $30 \mathrm{~min}$

A solution of $22(7.8 \mathrm{mg}, 0.048 \mathrm{mmol}, 1.0 \mathrm{eq})$ in THF $(0.9 \mathrm{~mL})$ was cooled to $-78{ }^{\circ} \mathrm{C}$ and KHMDS $(0.5 \mathrm{M}$ in PhMe, $0.045 \mathrm{~mL}, 0.023 \mathrm{mmol}, 1.25 \mathrm{eq}$ ) was added dropwise over the side of the flask. After stirring for $15 \mathrm{~min}$, Davis' oxaziridine ( $24 \mathrm{mg}, 0.091 \mathrm{mmol}, 5.0 \mathrm{eq}$ ) was added in one portion and the mixture was stirred for $20 \mathrm{~min}$ at $-78{ }^{\circ} \mathrm{C}$ and $10 \mathrm{~min}$ at room temperature. The mixture was transferred to a separation funnel and extracted with $\mathrm{Et}_{2} \mathrm{O}(3 \times 10 \mathrm{~mL})$. The organic phases were combined, dried over $\mathrm{Na}_{2} \mathrm{SO}_{4}$, filtered over a plug of $\mathrm{SiO}_{2}$ and concentrated under reduced pressure. The crude product was used without further purification in the next step. 


\section{Supporting Information}
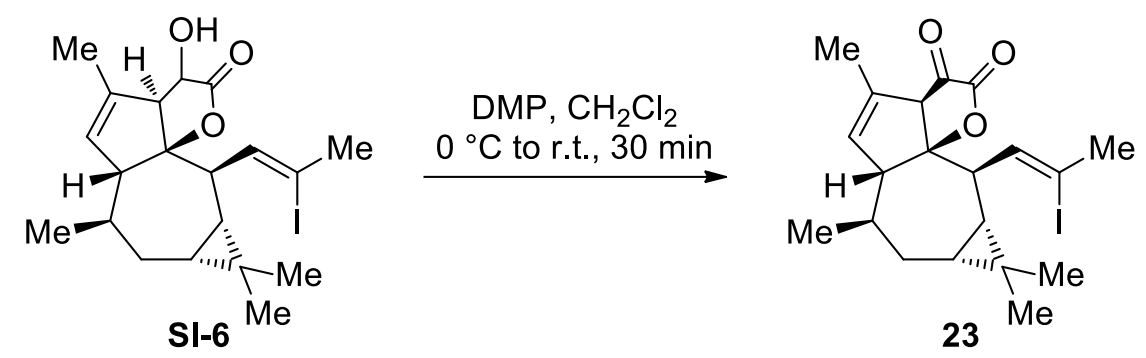

To a solution of crude $\mathrm{SI}-6$ in $\mathrm{CH}_{2} \mathrm{Cl}_{2}$ was added DMP (15 mg, $0.036 \mathrm{mmol}, 2.0$ eq) at $0{ }^{\circ} \mathrm{C}$. The mixture was warmed to room temperature and stirred for $30 \mathrm{~min}$. After completion of the reaction sat. aq. $\mathrm{NaHCO}_{3}(2 \mathrm{~mL})$ and sat. aq. $\mathrm{Na}_{2} \mathrm{SO}_{3}(2 \mathrm{~mL})$ was added, and the mixture was stirred for $5 \mathrm{~min}$. The mixture was added to a separation funnel and extracted with $\mathrm{Et}_{2} \mathrm{O}(3 \times 10 \mathrm{~mL})$. The organic phases were combined, dried over $\mathrm{Na}_{2} \mathrm{SO}_{4}$, filtered and concentrated under reduced pressure. The crude product was purified by flash column chromatography (hexane-Et $\mathrm{E}_{2} \mathrm{O}, 95: 5$ ) to give $\mathbf{2 3}$ as a yellowish oil (3.9 mg, $0.0088 \mathrm{mmol}, 49 \%$ ).

It has to be noted that $\mathbf{2 3}$ was contaminated with impurities generated during vinyl iodide formation step, which were not separated at this point and amount to maximally about $5 \%$ total. They include dimethyl derivative, protodehalogenated, derivative, and vinyl bromide. The product was carried as is into the next step.

${ }^{1} \mathrm{H}-\mathrm{NMR}\left(400 \mathrm{MHz}, \mathrm{CDCl}_{3}\right) \delta(500 \mathrm{MHz}$, Chloroform-d) $\delta 5.73$ (td, $J=2.6,1.5 \mathrm{~Hz}, 1 \mathrm{H}), 5.29$ (dd, J = 9.1, $1.5 \mathrm{~Hz}, 1 \mathrm{H}), 3.60(\mathrm{dq}, J=2.5,1.3 \mathrm{~Hz}, 1 \mathrm{H}), 2.91-2.85(\mathrm{~m}, 1 \mathrm{H}), 2.68(\mathrm{dd}, J=10.5,9.2 \mathrm{~Hz}, 1 \mathrm{H}), 2.46(\mathrm{~d}, J=$ $1.5 \mathrm{~Hz}, 3 \mathrm{H}), 1.93-1.86(\mathrm{~m}, 1 \mathrm{H}), 1.79-1.74(\mathrm{~m}, 1 \mathrm{H}), 1.67(\mathrm{q}, J=1.6 \mathrm{~Hz}, 3 \mathrm{H}), 1.07(\mathrm{~s}, 3 \mathrm{H}), 1.04(\mathrm{~s}, 3 \mathrm{H})$, $0.96(\mathrm{~d}, J=6.6 \mathrm{~Hz}, 3 \mathrm{H}), 0.89-0.87(\mathrm{~m}, 1 \mathrm{H}), 0.82-0.79(\mathrm{~m}, 1 \mathrm{H})$.

${ }^{13}$ C-NMR: $\left(101 \mathrm{MHz}, \mathrm{CDCl}_{3}\right) \delta(126 \mathrm{MHz}$, Chloroform-d) $\delta$ 189.7,160.3, 134.3, 133.5, 125.7, 107.2, 61.1, $57.4,48.7,34.2,33.6,32.9,30.5,28.6,21.4,21.3,19.3,16.9,15.4,14.0$.

IR (thin film, cm ${ }^{-1}$ ): 2954, 2925, 1789, 1784, 1481, 1390, 1242.

HRMS (ESI): $m / z$ : exact mass calculated for $\mathrm{C}_{20} \mathrm{H}_{25} \mathrm{NaO}_{3}[\mathrm{M}+\mathrm{Na}]^{+}, 463.0741$; found: 463.0738 .

$[\mathrm{a}]_{\mathrm{D}}^{25^{\circ}}=-3.7\left(\mathrm{c}=0.2, \mathrm{CHCl}_{3}\right)$. 


\section{Supporting Information}

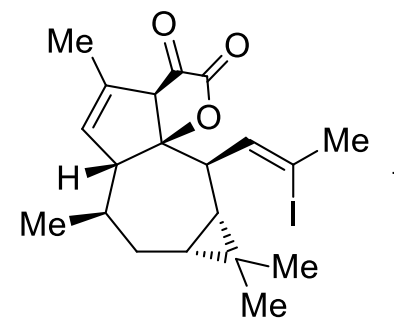

23

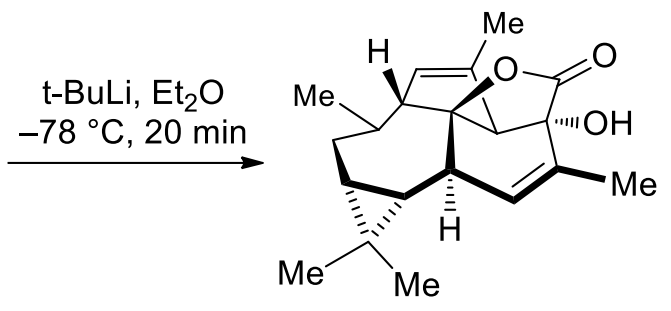

Euphorikanin A (1)

To a solution of $t$-BuLi (1.7 $\mathrm{M}$ in pentane, $20 \mu \mathrm{L}, 0.034 \mathrm{mmol}, 3.0$ eq) in $\mathrm{Et}_{2} \mathrm{O}(0.4 \mathrm{~mL})$ was added ketolactone $23(3.9 \mathrm{mg}, 0.0088 \mathrm{mmol}, 1.0 \mathrm{eq})$ in $\mathrm{Et}_{2} \mathrm{O}(0.5 \mathrm{~mL})$ at $-78^{\circ} \mathrm{C}$. The mixture was stirred for $30 \mathrm{~min}$ and quenched by the addition of sat. aq. $\mathrm{NH}_{4} \mathrm{Cl}(2 \mathrm{~mL})$ at the same temperature. After warming to room temperature, the mixture was transferred to a separation funnel and extracted with EtOAc ( 3 $x 10 \mathrm{~mL}$ ). the organic phases were dried over $\mathrm{Na}_{2} \mathrm{SO}_{4}$, filtered and concentrated und reduced pressure. The crude product was purified by flash column chromatography (hexane-EtOAc, 70:30) to give 1 as a colorless solid (1.1 mg, $3.54 \mu \mathrm{mol}, 40 \%$ ).

${ }^{1} \mathrm{H}-\mathrm{NMR}(600 \mathrm{MHz}$, Chloroform-d) $\delta 5.53(\mathrm{tt}, J=3.2,1.6 \mathrm{~Hz}, 1 \mathrm{H}$ ), 5.39 (dq, J = 3.2, 1.6 Hz, 1H), 3.09 (dt, $J=2.4,1.3 \mathrm{~Hz}, 1 \mathrm{H}), 2.71(\mathrm{~m}, 2 \mathrm{H}), 2.34(\mathrm{dt}, J=11.4,2.4 \mathrm{~Hz}, 1 \mathrm{H}), 1.84(\mathrm{q}, J=1.7 \mathrm{~Hz}, 3 \mathrm{H}), 1.81(\mathrm{dd}, J=2.3$, $1.6 \mathrm{~Hz}, 3 \mathrm{H}), 1.79(\mathrm{dd}, J=6.2,3.7 \mathrm{~Hz}, 1 \mathrm{H}), 1.72-1.68(\mathrm{~m}, 1 \mathrm{H}), 1.34(\mathrm{~m}, 2 \mathrm{H}), 1.05(\mathrm{~s}, 3 \mathrm{H}), 1.00(\mathrm{~s}, 3 \mathrm{H})$, $0.88(\mathrm{~s}, 3 \mathrm{H}), 0.82-0.79(\mathrm{~m}, 1 \mathrm{H}), 0.69(\mathrm{td}, J=9.7,3.6 \mathrm{~Hz}, 1 \mathrm{H})$.

${ }^{13}$ C-NMR: (151 MHz, Chloroform-d) $\delta$ 130.6, 126.1, 59.7, 54.6, 39.1, 32.7, 31.9, 31.9, 31.3, 29.7, 28.6, 26.6, 20.9, 18.8, 16.6, 16.2, 15.3, 1.0.

IR (thin film, $\mathrm{cm}^{-1}$ ): 3442, 2923, 2854, 1758, 1665, 1447, 1378, 1260, 1211, 1182, 1158, 1146, 1084, $1012,954,929,881,838,800,725,708,685,660,635,521,497$.

HRMS (ESI): $m / z$ : exact mass calculated for $\mathrm{C}_{20} \mathrm{H}_{26} \mathrm{NaO}_{3}[\mathrm{M}+\mathrm{Na}]^{+}, 337.1774$; found: 337.1773 .

$[\mathrm{a}]_{\mathrm{D}}{ }^{25^{\circ}}=+6\left(\mathrm{c}=0.02, \mathrm{CHCl}_{3}\right)$. 
NMR Spectra 


\section{Supporting Information}

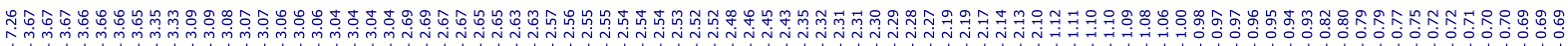

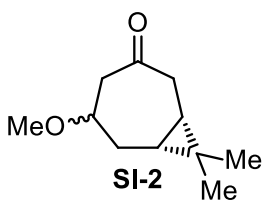

$400 \mathrm{MHz}, \mathrm{CDCl}_{3}$

ڤั่

$\mathrm{CHCl}_{3}$

Wullowith

Whith
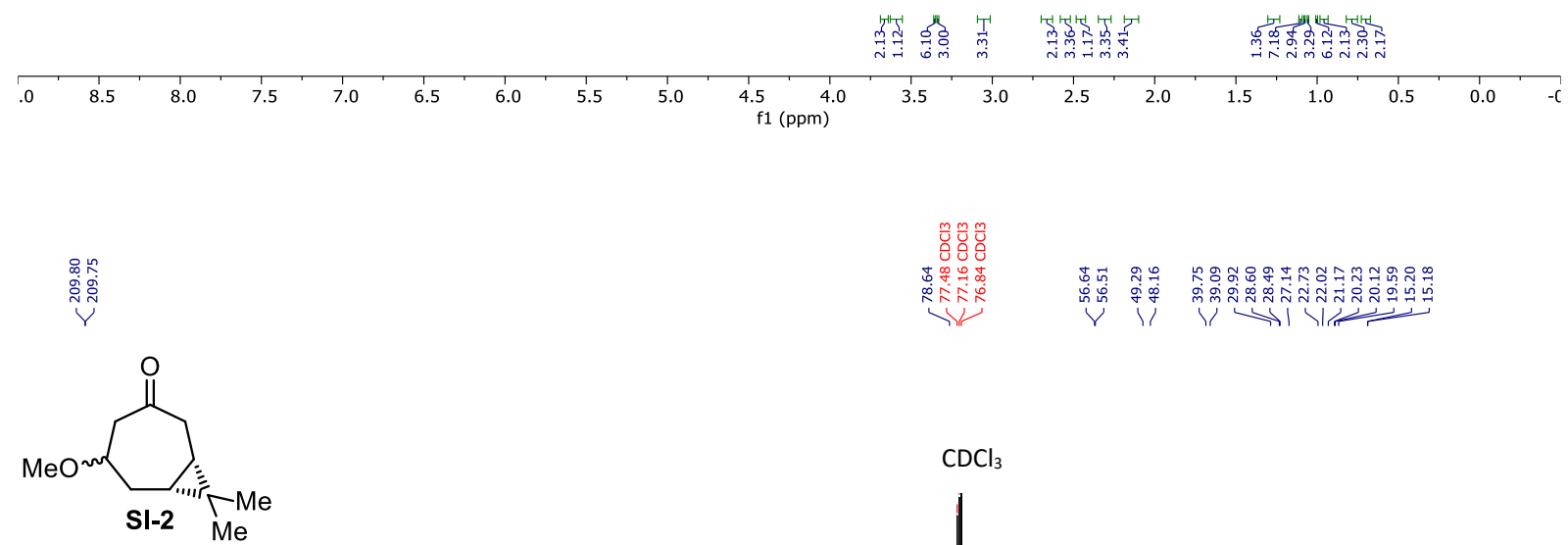

$\underbrace{\underbrace{}_{0}}$

$101 \mathrm{MHz}, \mathrm{CDCl}_{3}$

$\begin{array}{llllllllllllllllllllllllllllllllll}20 & 210 & 200 & 190 & 180 & 170 & 160 & 150 & 140 & 130 & 120 & 110 & 100 & 90 & 80 & 70 & 60 & 50 & 40 & 30 & 20 & 10 & 0 & -10\end{array}$

$\mathrm{CDCl}_{3}$

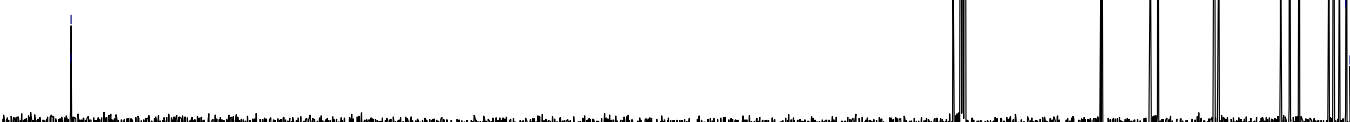




\section{Supporting Information}

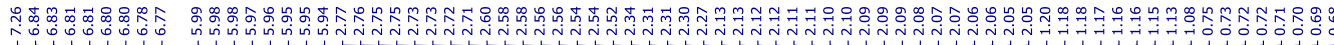

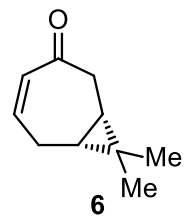

$400 \mathrm{MHz}, \mathrm{CDCl}_{3}$
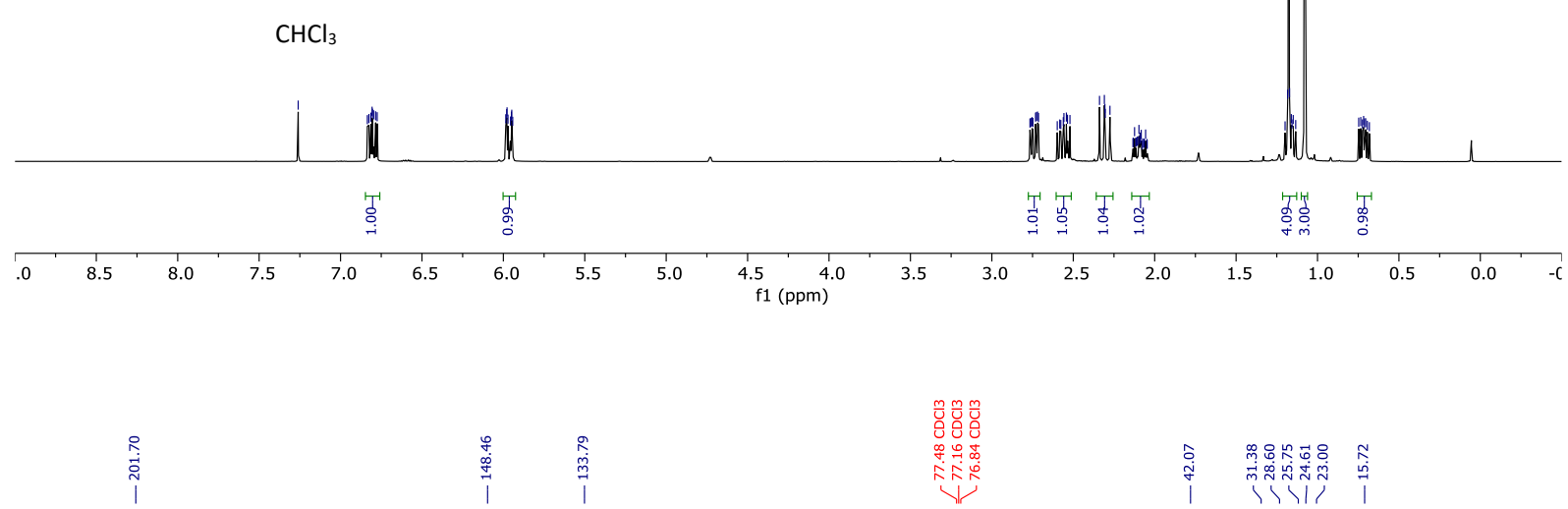

啇

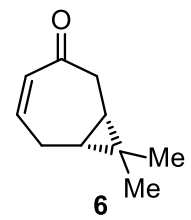

$101 \mathrm{MHz}, \mathrm{CDCl}_{3}$

$\begin{array}{lllllllllllll}20 & 210 & 200 & 190 & 180 & 170 & 160 & 150 & 140 & 130 & 120 & 110 & 100\end{array}$

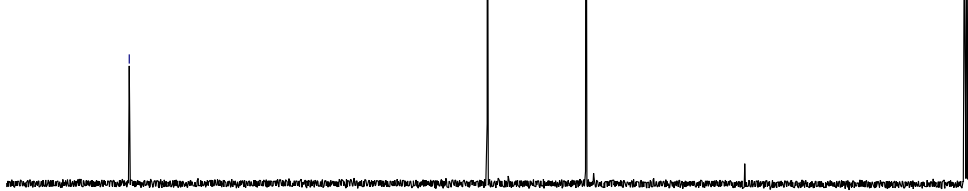

$\mathrm{DCl}$
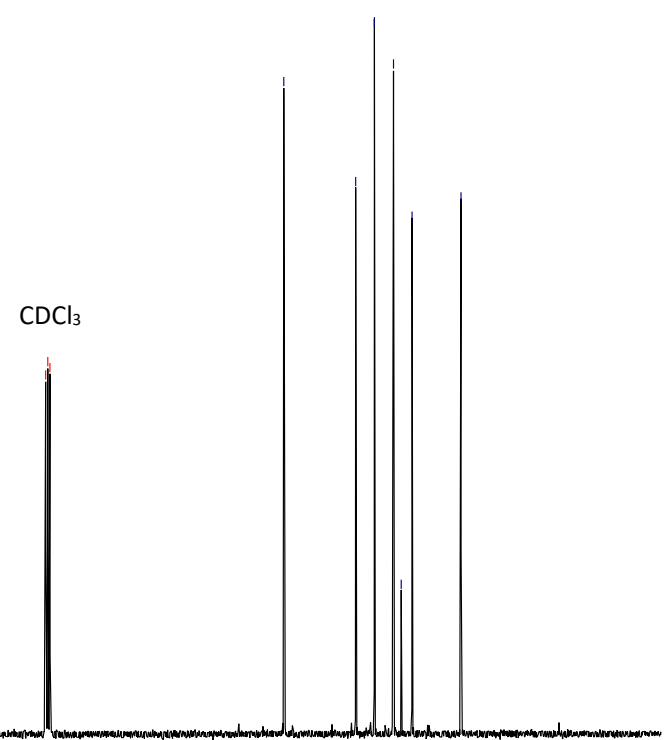


\section{Supporting Information}
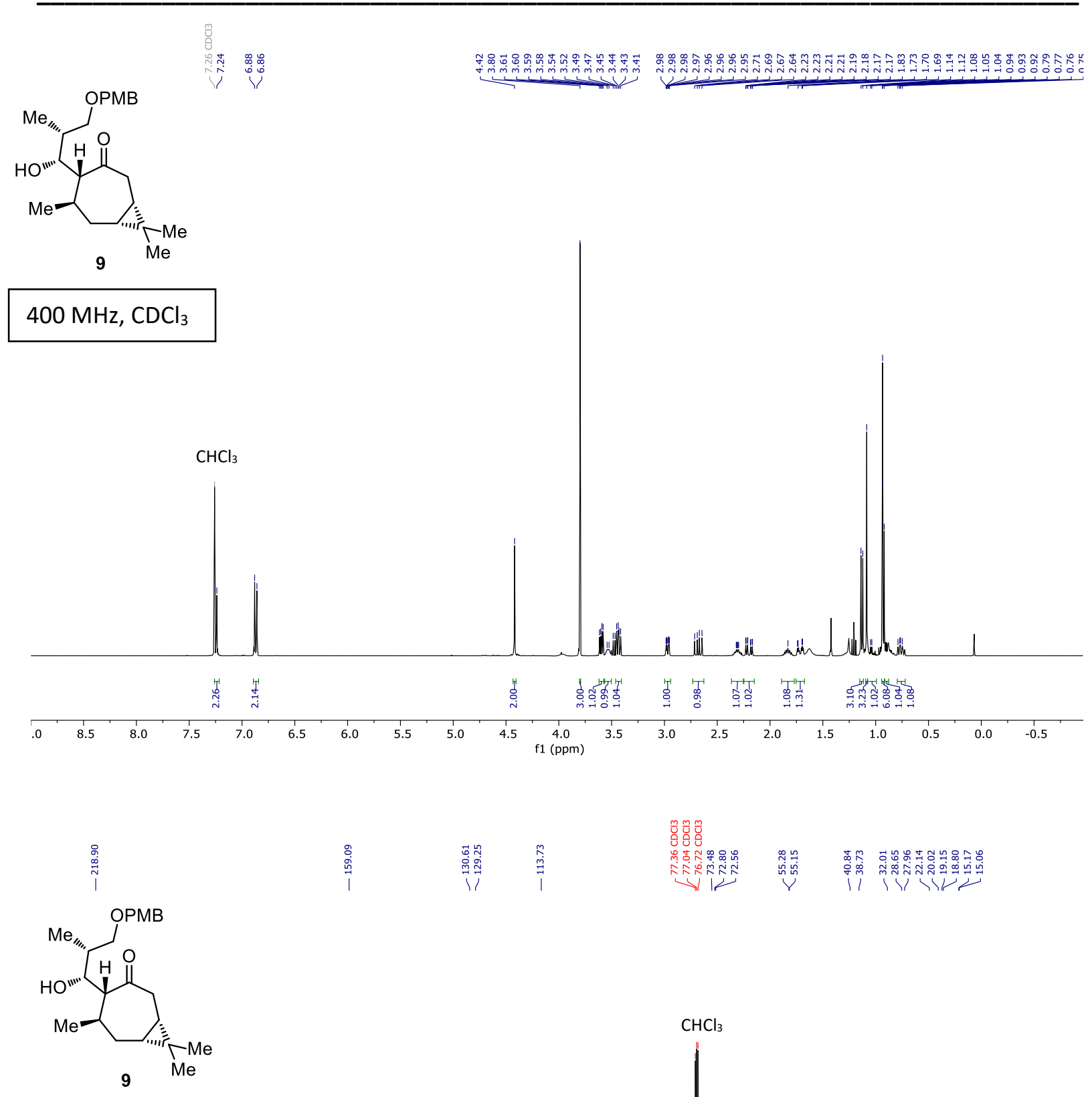

$\mathrm{CHCl}_{3}$

$101 \mathrm{MHz}, \mathrm{CDCl}_{3}$

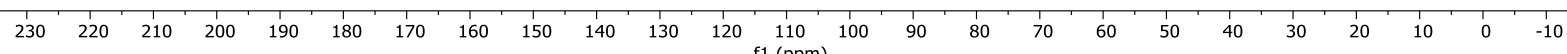




\section{Supporting Information}

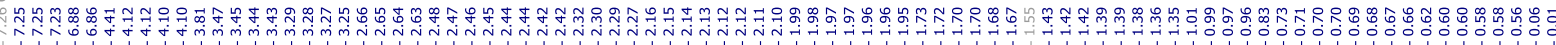

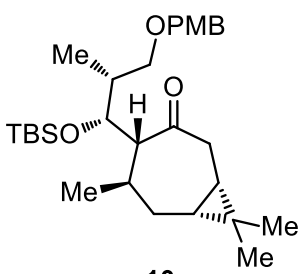

10

$400 \mathrm{MHz}^{\mathrm{CDCl}_{3}}$
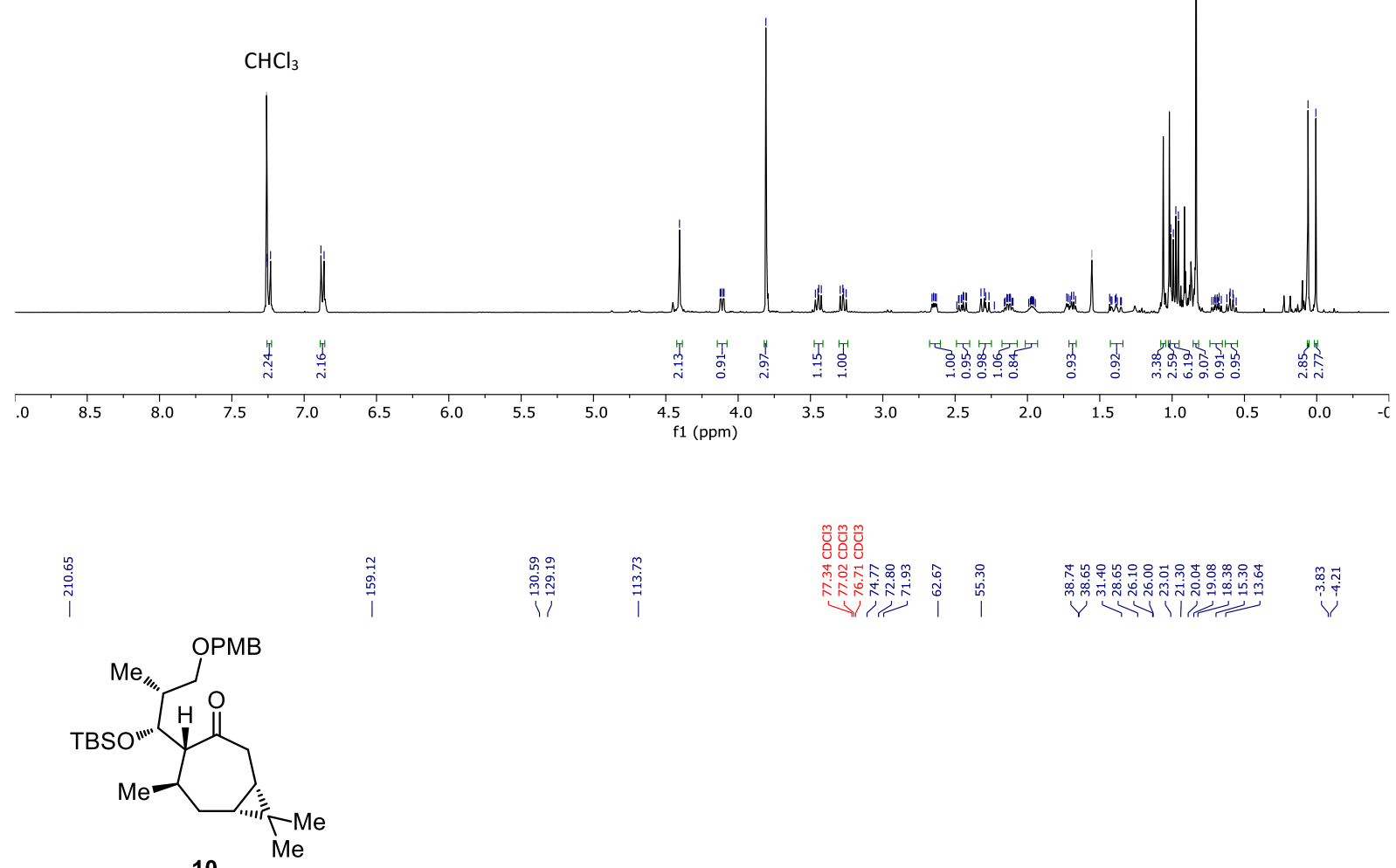

10

$\mathrm{CDCl}_{3}$

$101 \mathrm{MHz}^{\mathrm{CDCl}_{3}}$

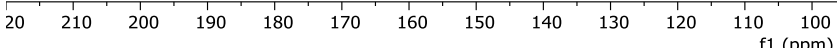


0

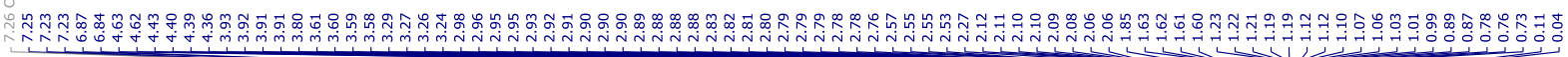

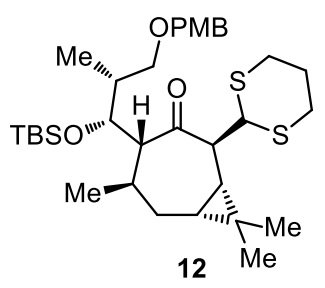

$400 \mathrm{MHz}, \mathrm{CDCl}_{3}$
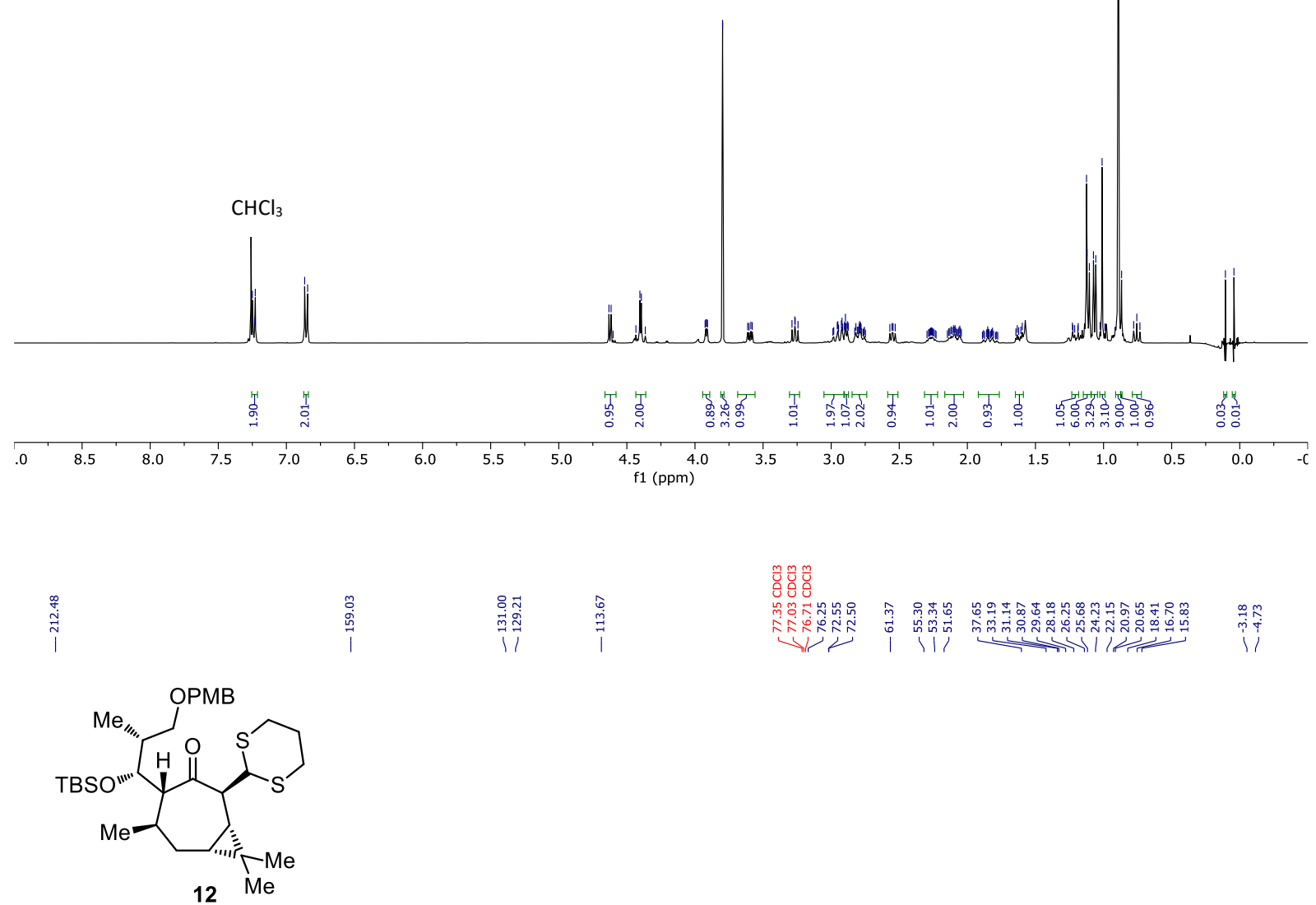

$101 \mathrm{MHz}, \mathrm{CDCl}_{3}$

$\mathrm{CDCl}_{3}$

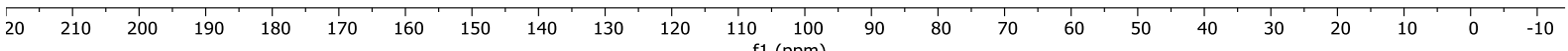




\section{Supporting Information}

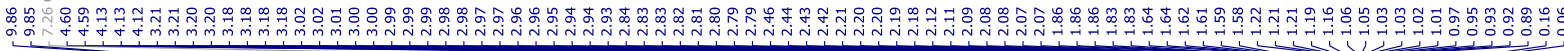

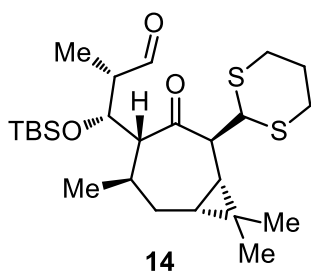

$400 \mathrm{MHz}, \mathrm{CDCl}_{3}$

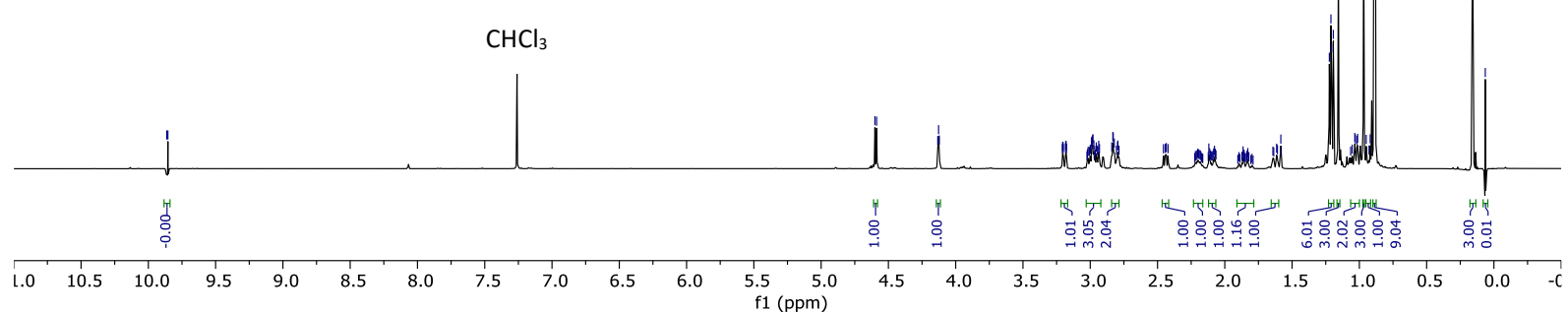

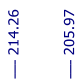

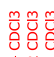

急

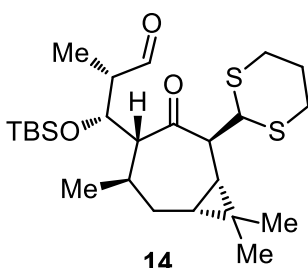

$\mathrm{CDCl}_{3}$

$101 \mathrm{MHz}, \mathrm{CDCl}_{3}$

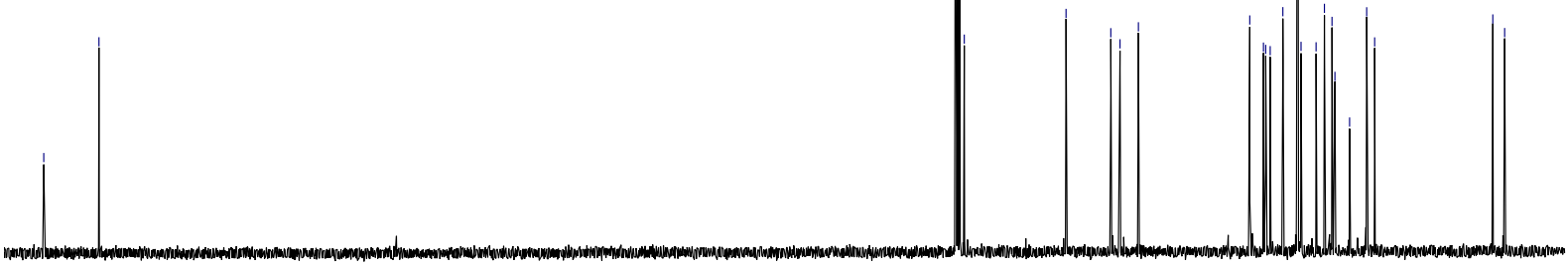

$\begin{array}{lllllllllllllllllllllllll}1 & 210 & 200 & 190 & 180 & 170 & 160 & 150 & 140 & 130 & 120 & 110 & 100 & 90 & 80 & 70 & 60 & 50 & 40 & 30 & 20 & 10 & 0 & -10\end{array}$ 


\section{Supporting Information}

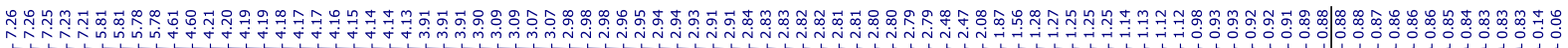

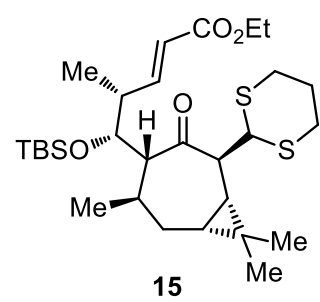

$500 \mathrm{MHz}, \mathrm{CDCl}_{3}$
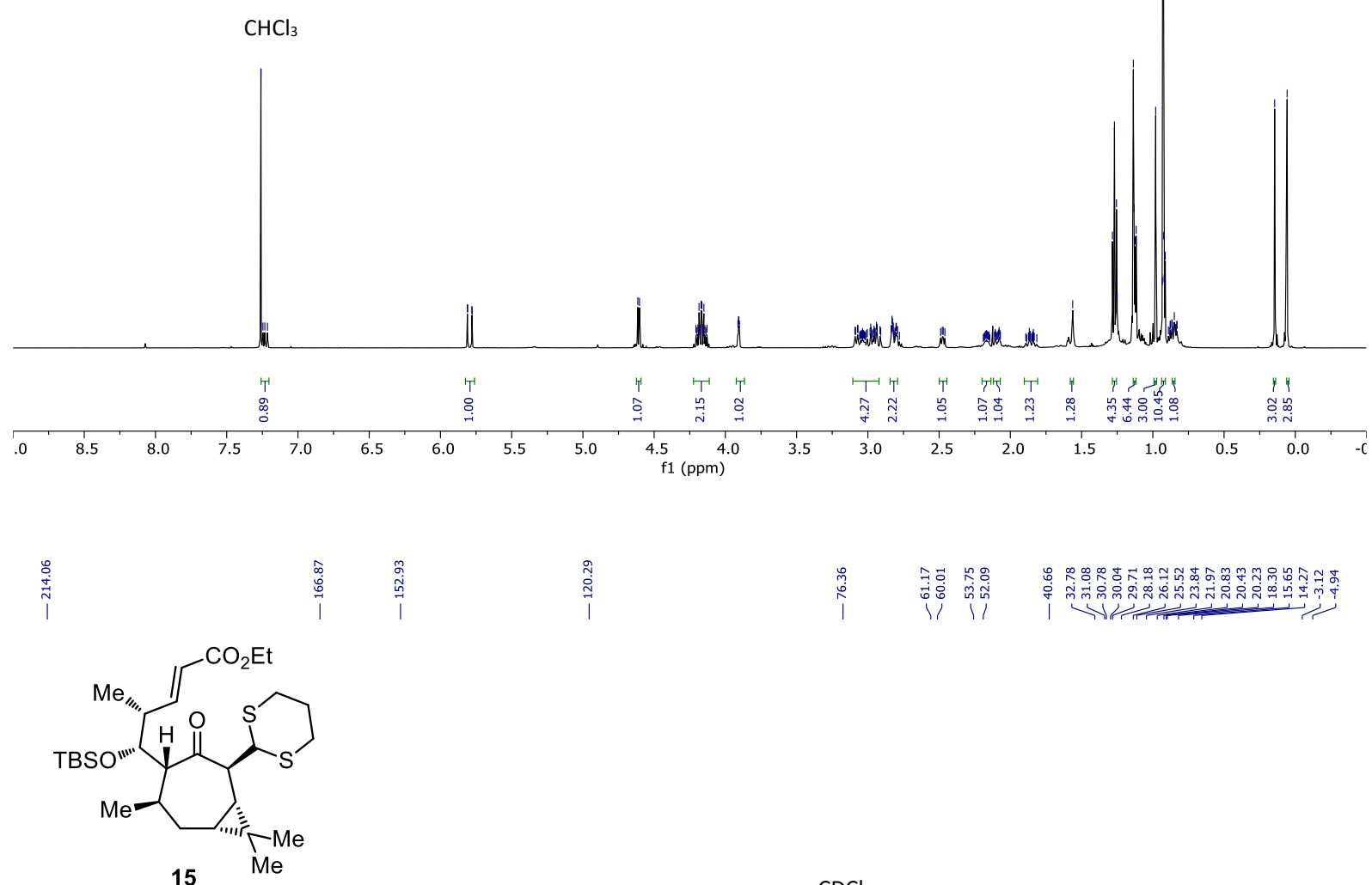

$126 \mathrm{MHz}, \mathrm{CDCl}_{3}$

$\mathrm{CDCl}_{3}$

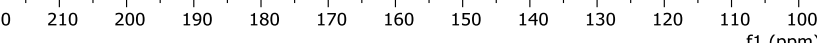




\section{Supporting Information}

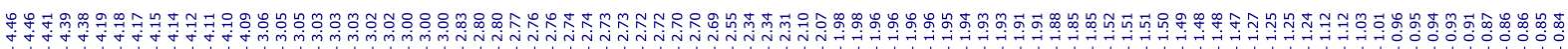

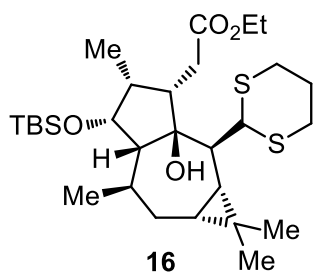

$500 \mathrm{MHz}, \mathrm{CDCl}_{3}$

Whe

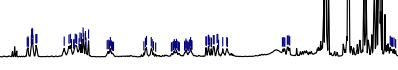
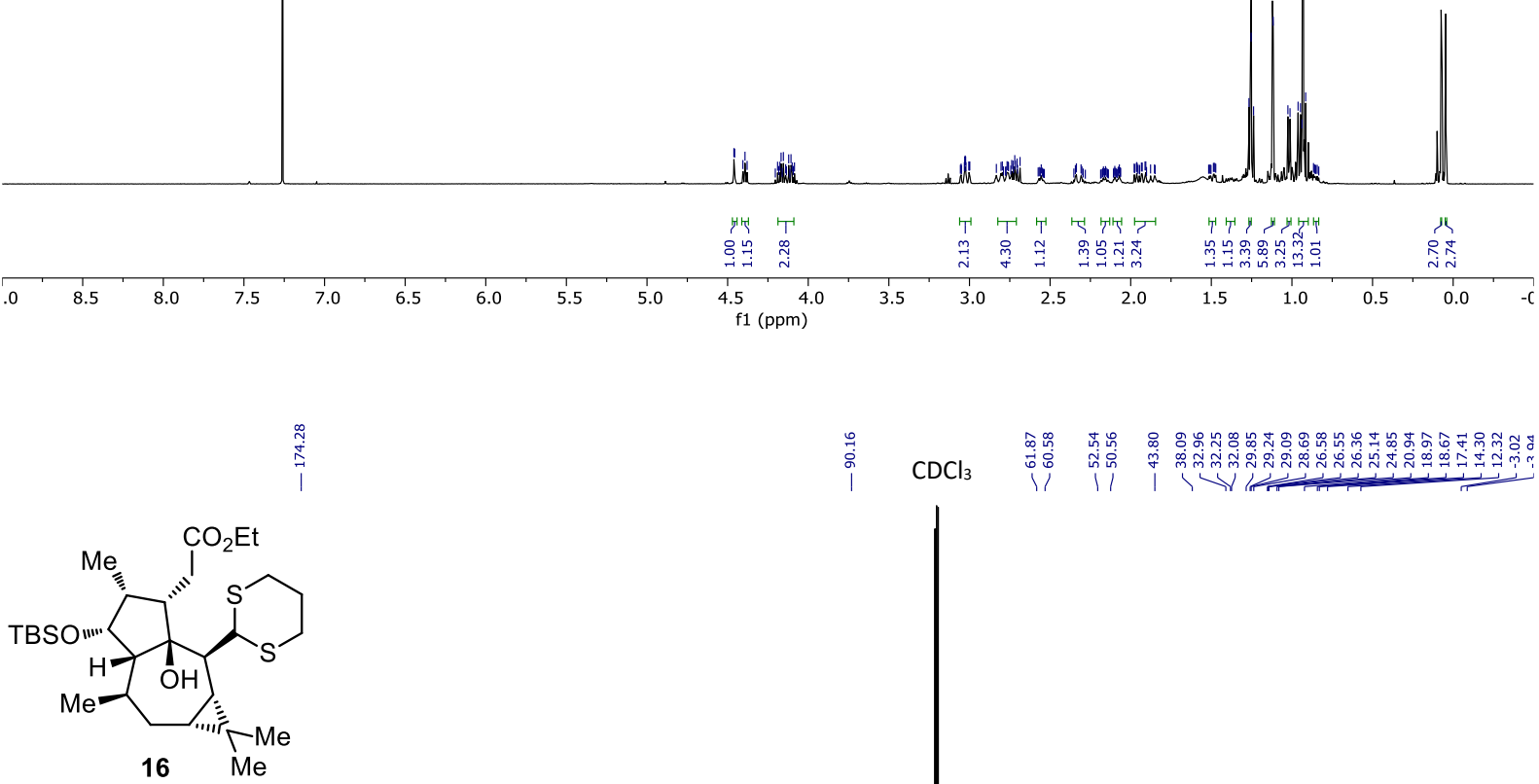

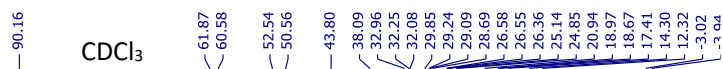

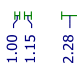

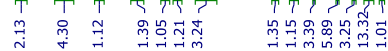
f1 (ppm)

$126 \mathrm{MHz}^{\mathrm{CDCl}_{3}}$

$\begin{array}{lllllllllllll}20 & 210 & 200 & 190 & 180 & 170 & 160 & 150 & 140 & 130 & 120 & 110 & 100 \\ \mathrm{f} 1(\mathrm{ppm})\end{array}$

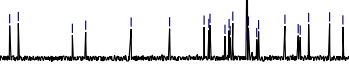




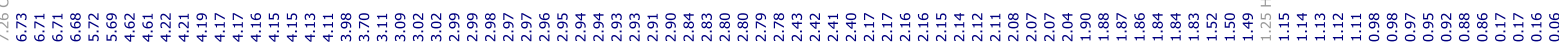

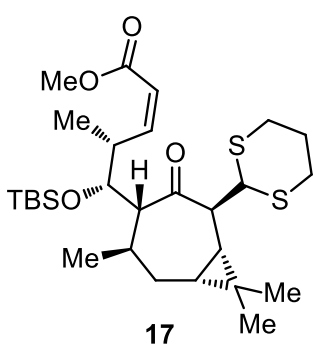

$400 \mathrm{MHz}^{\mathrm{CDCl}_{3}}$
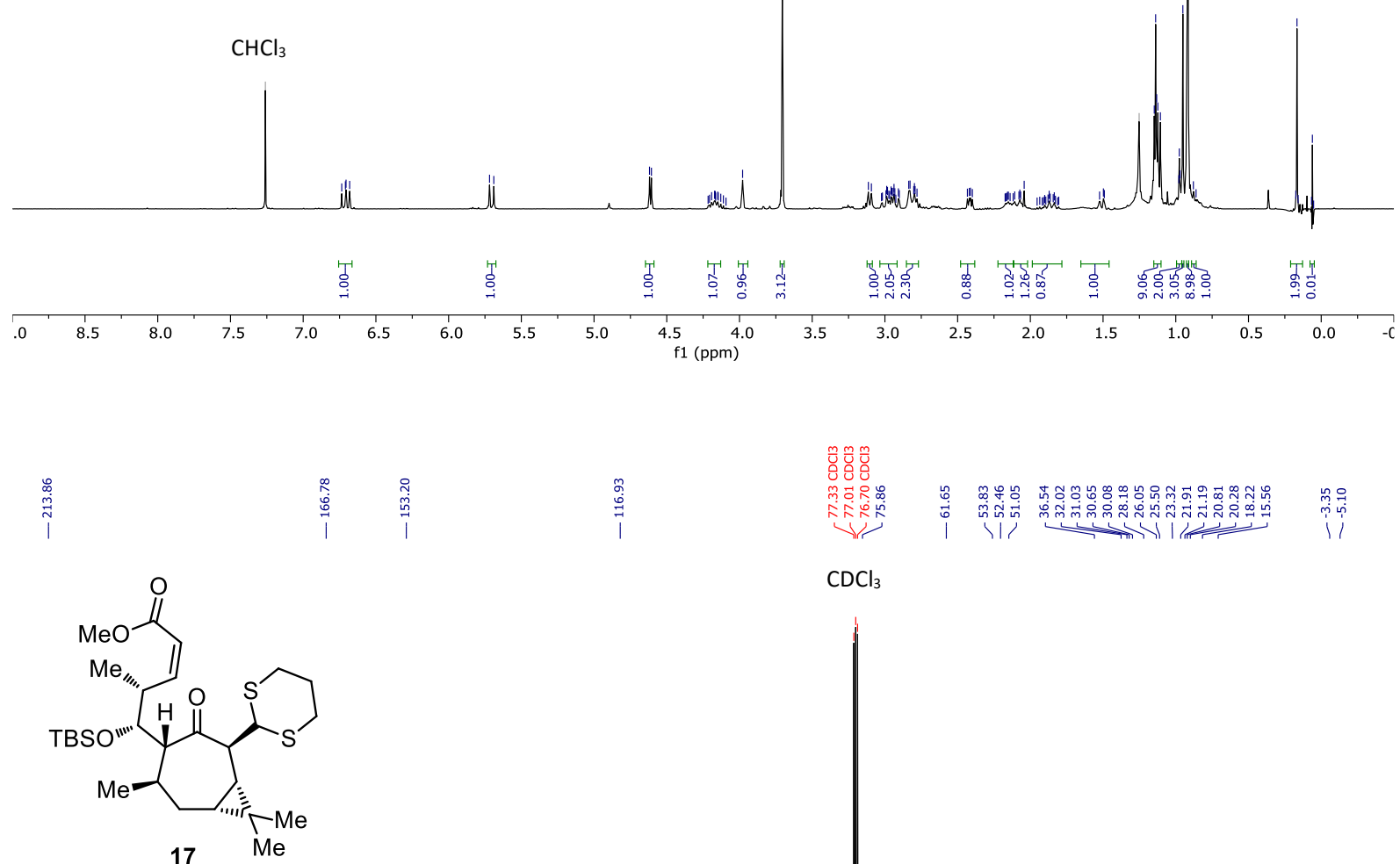

$101 \mathrm{MHz}, \mathrm{CDCl}_{3}$ 


\section{Supporting Information}

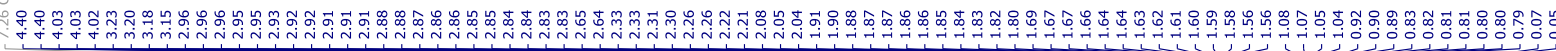

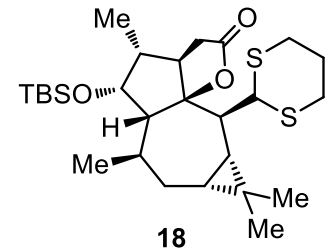

$400 \mathrm{MHz} \mathrm{CDCl}_{3}$
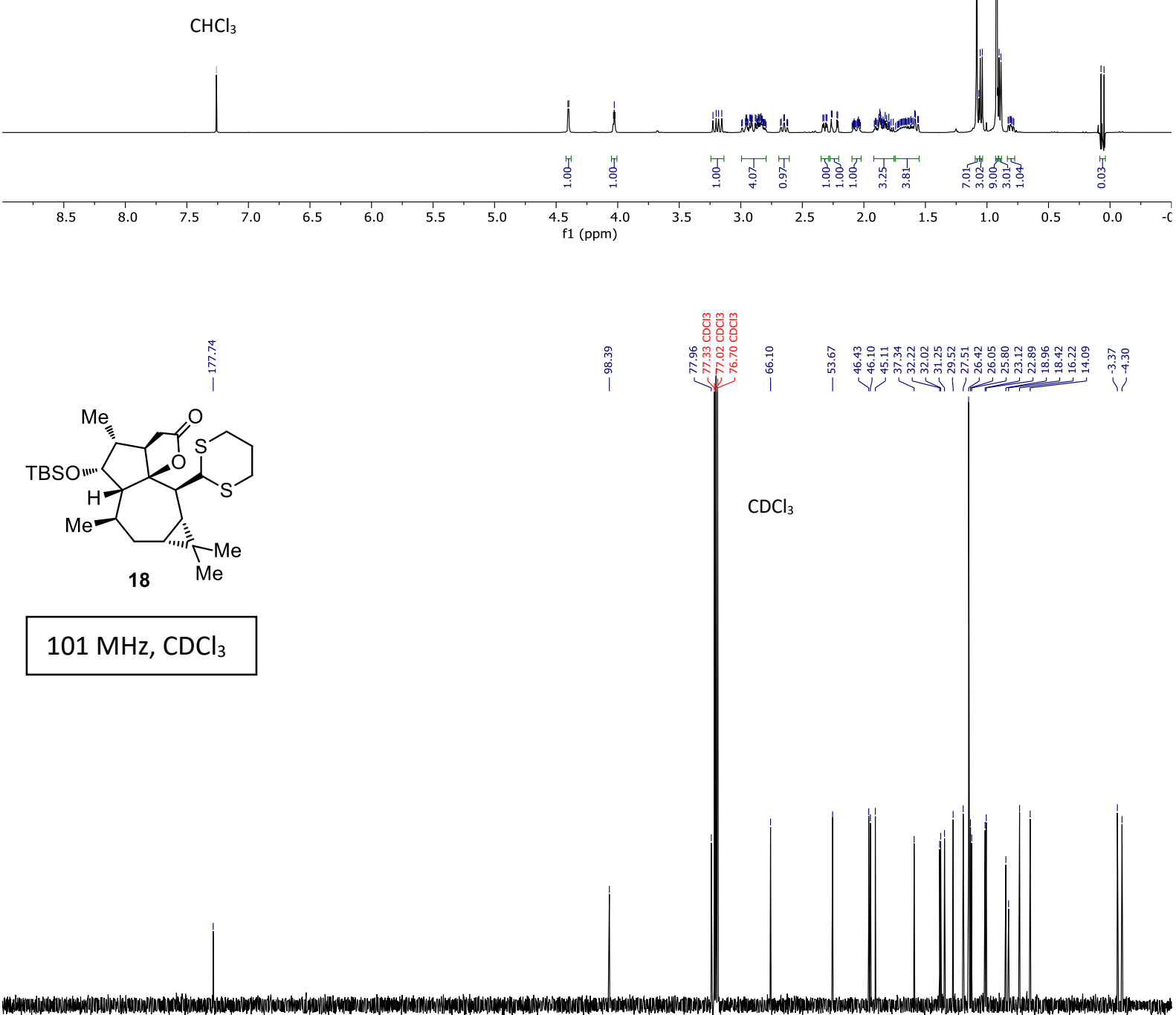

$101 \mathrm{MHz}, \mathrm{CDCl}_{3}$

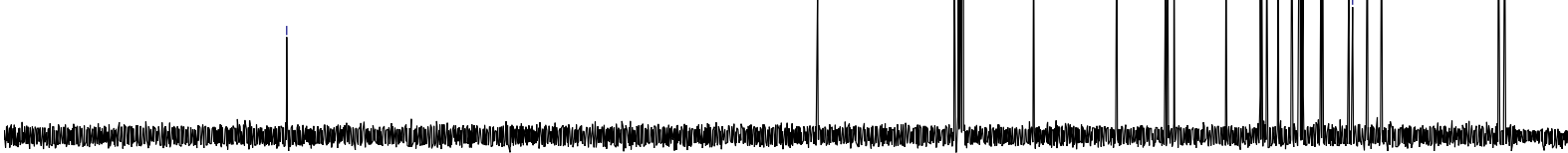

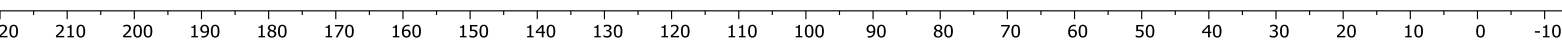
f1 (ppm) 


\section{Supporting Information}

Y

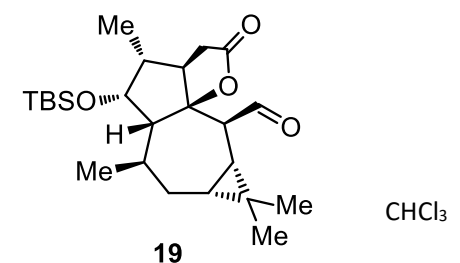

$$
400 \mathrm{MHz}, \mathrm{CDCl}_{3}
$$
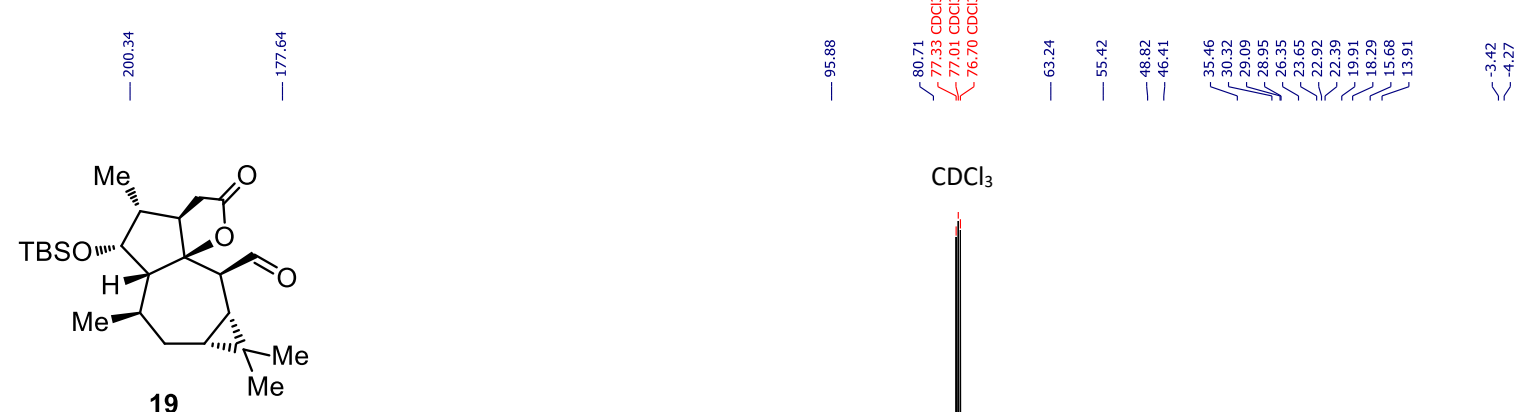

$\mathrm{CDCl}_{3}$

$101 \mathrm{MHz}^{\mathrm{CDCl}_{3}}$

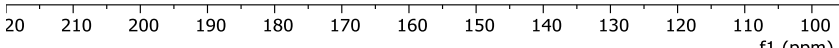

.
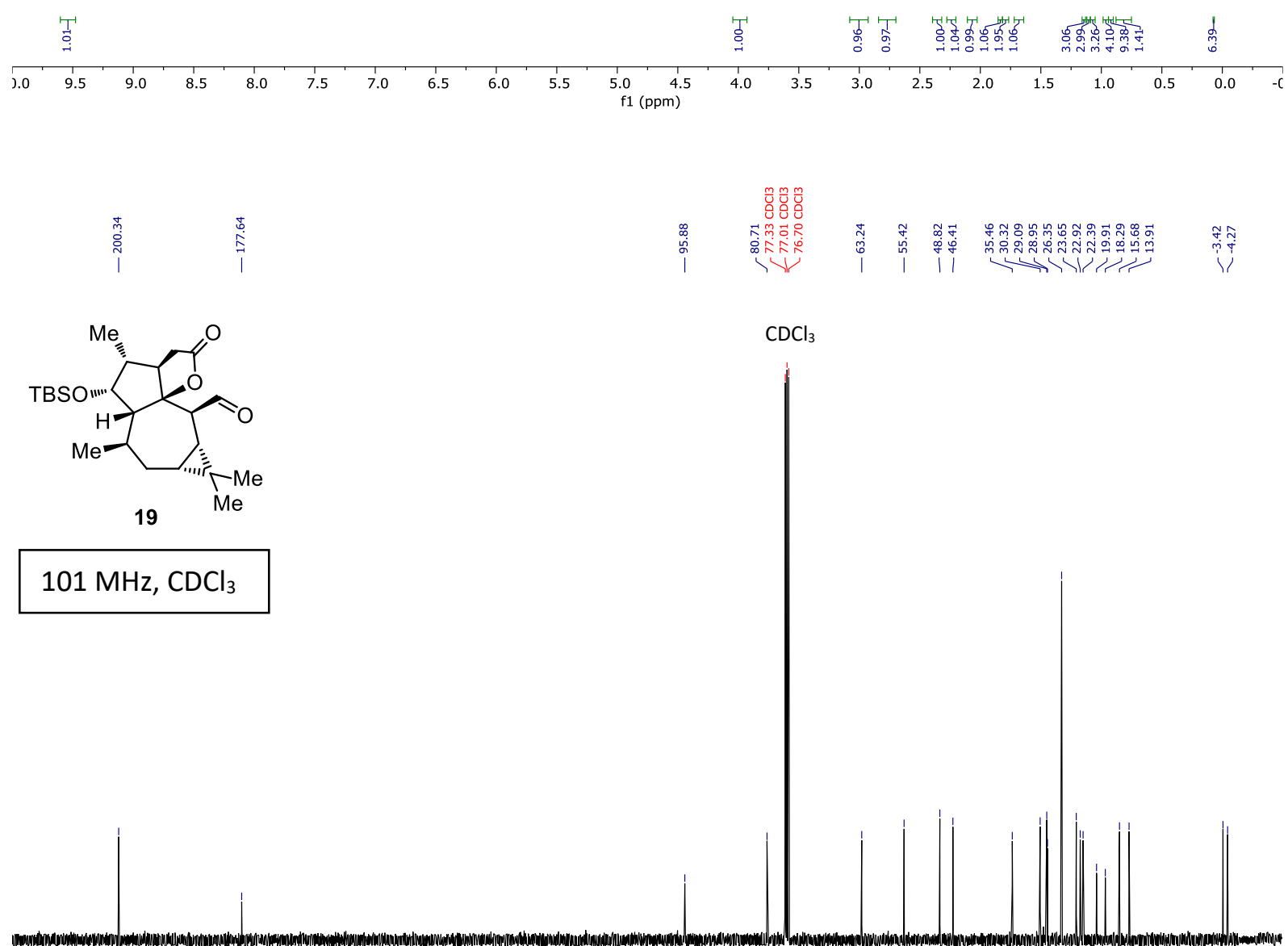

$\begin{array}{lllllllllllll}20 & 210 & 200 & 190 & 180 & 170 & 160 & 150 & 140 & 130 & 120 & 110 & 100 \\ & & & & & \end{array}$ 


\section{Supporting Information}
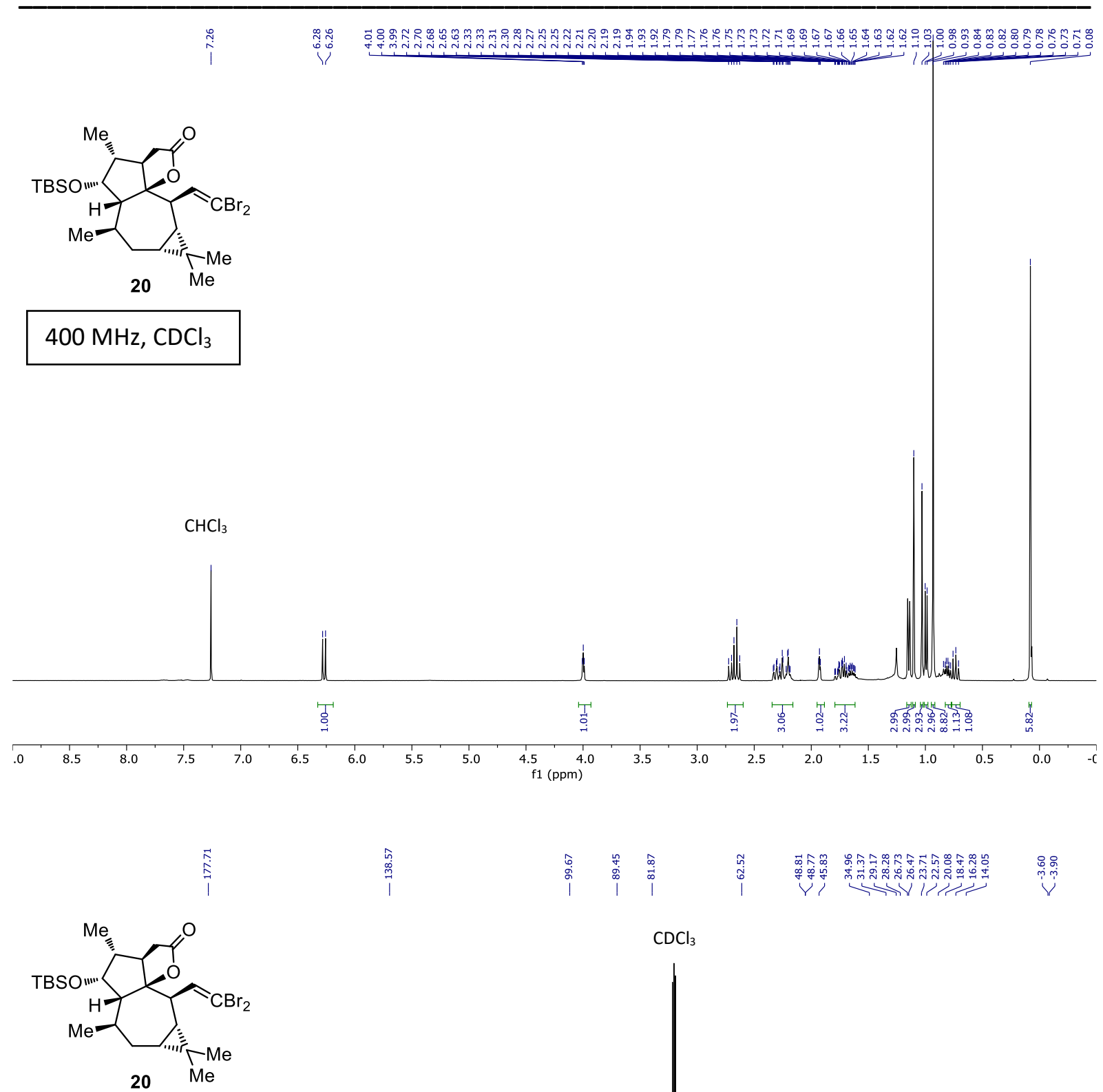

$\mathrm{CDCl}_{3}$

$101 \mathrm{MHz}, \mathrm{CDCl}_{3}$

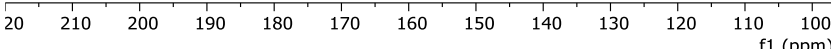




\section{Supporting Information}

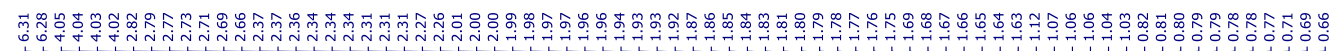

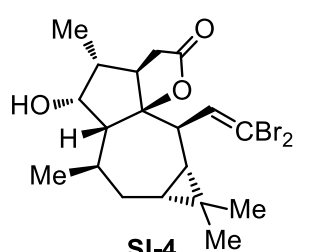

$400 \mathrm{MHz}, \mathrm{CDCl}_{3}$

$\mathrm{CHCl}_{3}$
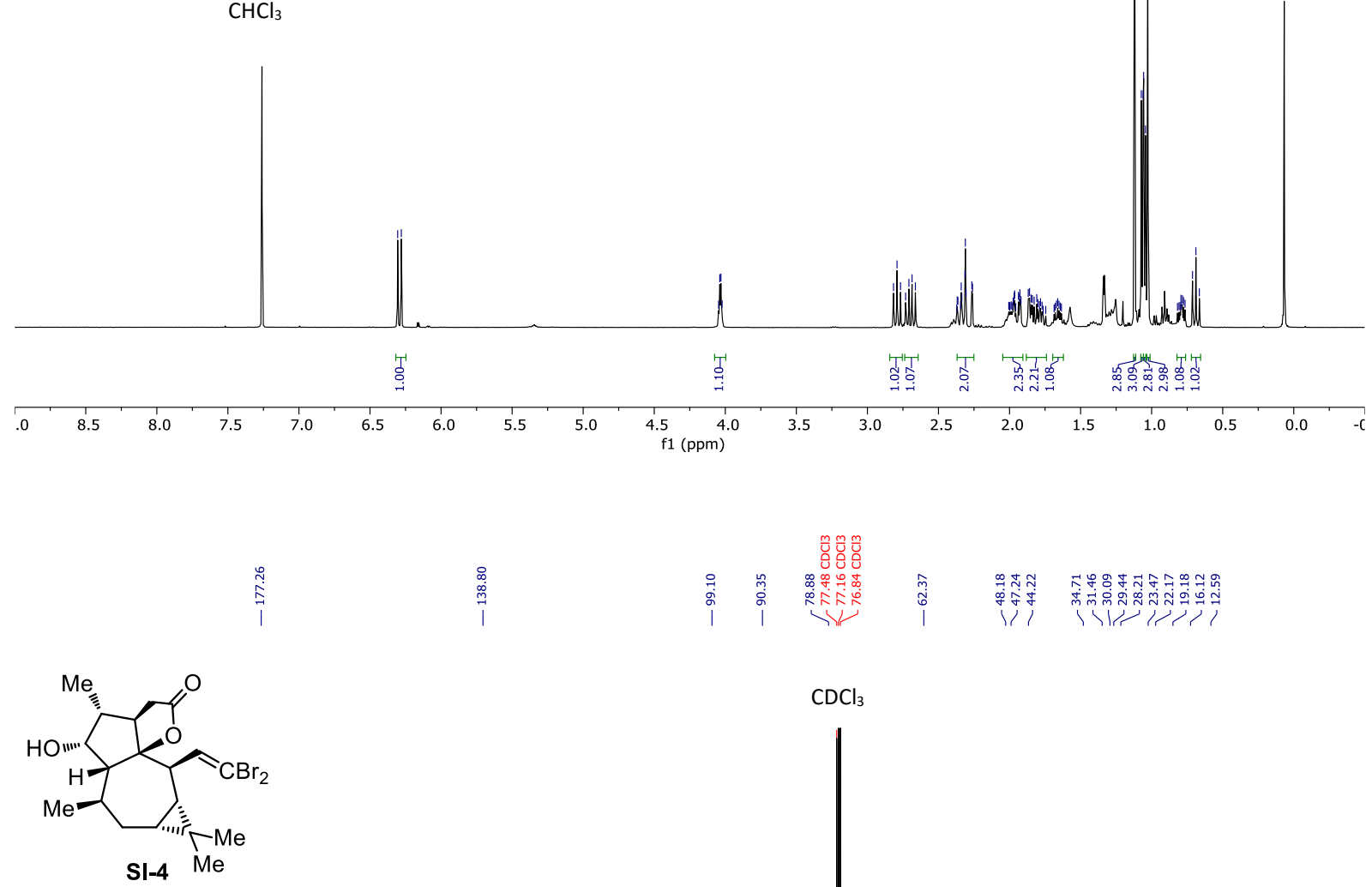

$\mathrm{CDCl}_{3}$

$101 \mathrm{MHz}^{\mathrm{CDCl}_{3}}$

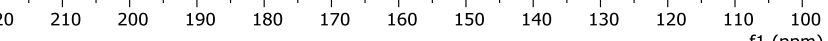




\section{Supporting Information}
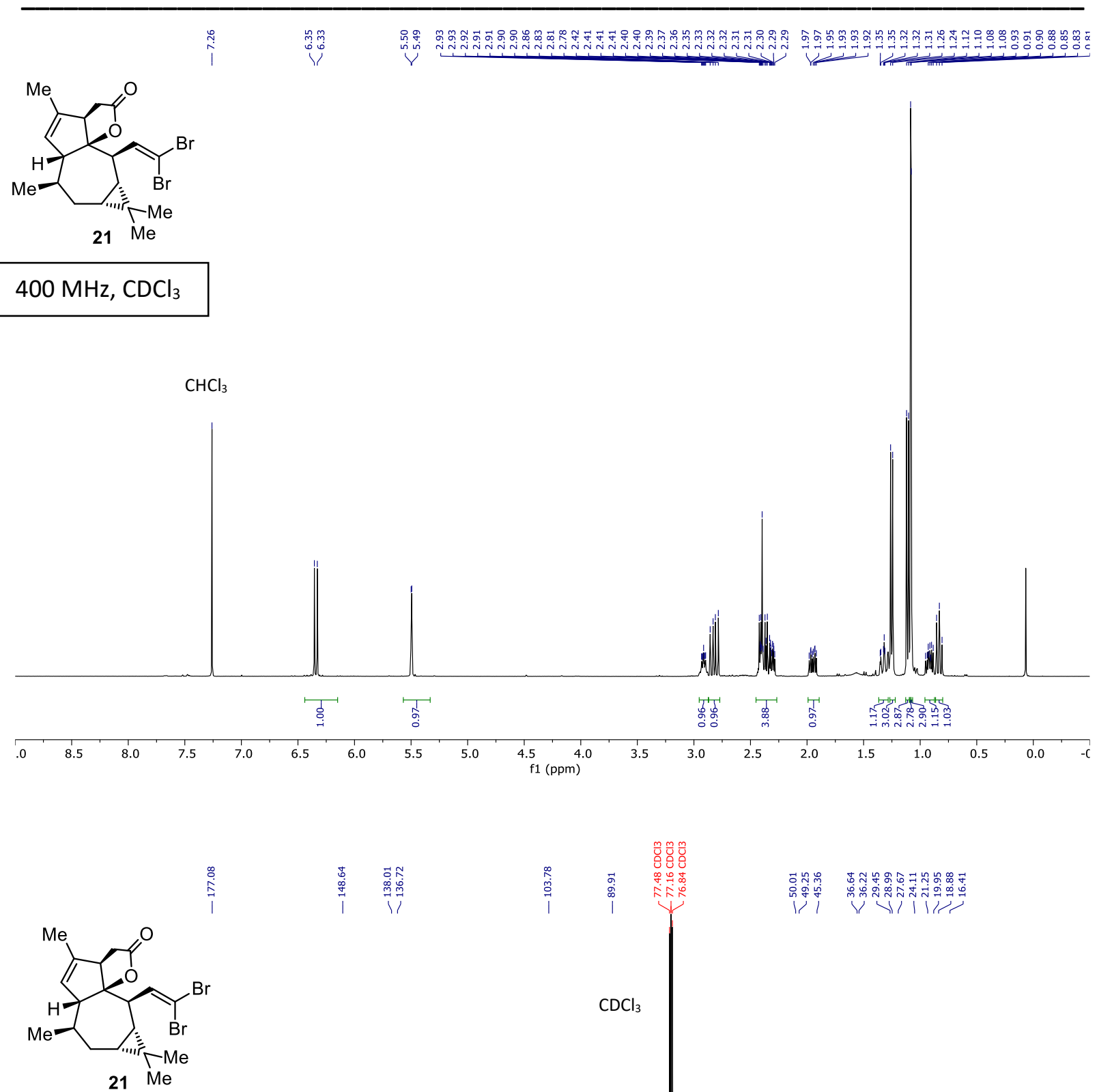

$101 \mathrm{MHz}, \mathrm{CDCl}_{3}$

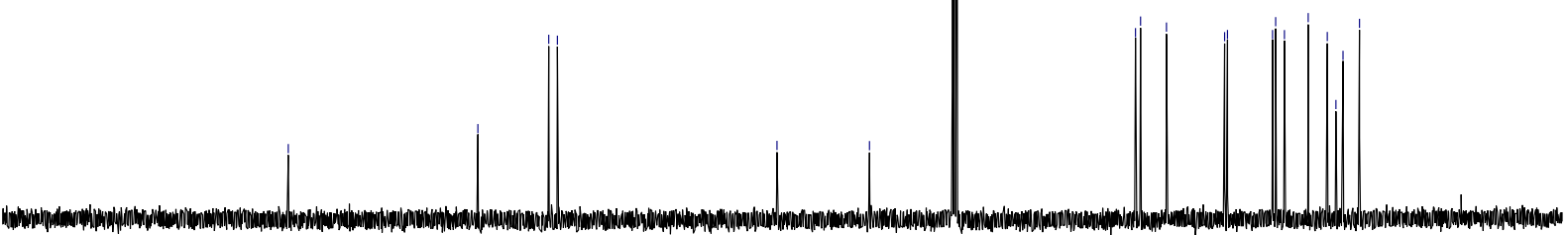

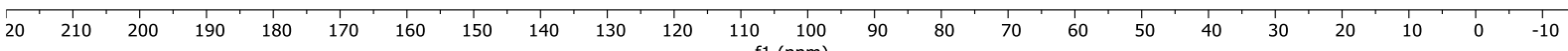
f1 (ppm) 


\section{Supporting Information}

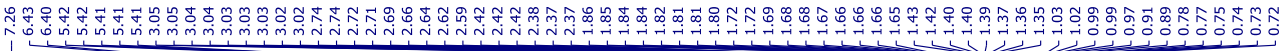

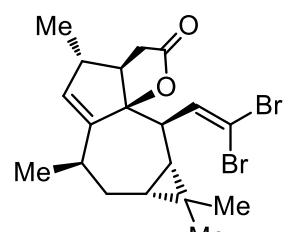

SI-5 Me
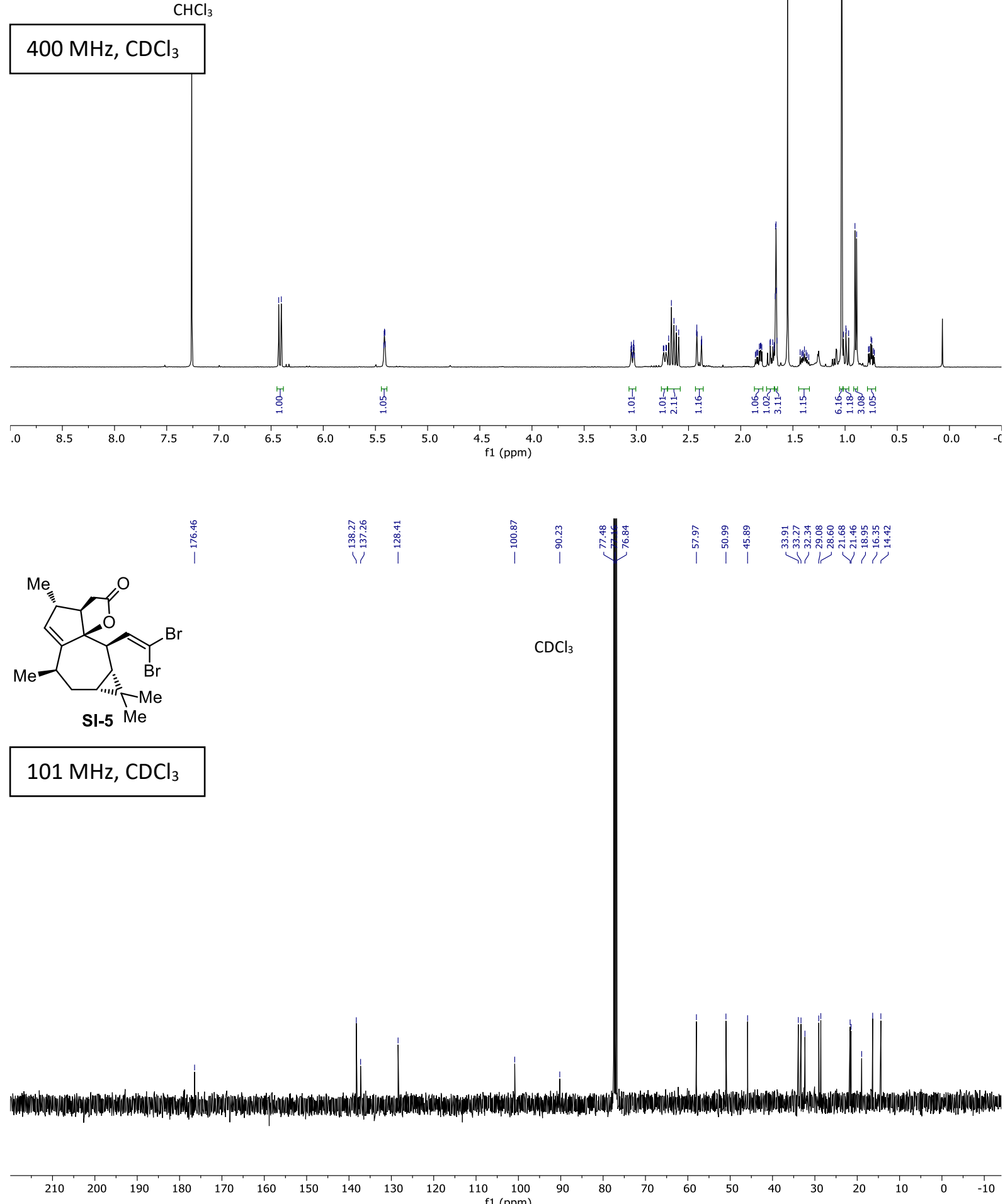


\section{Supporting Information}
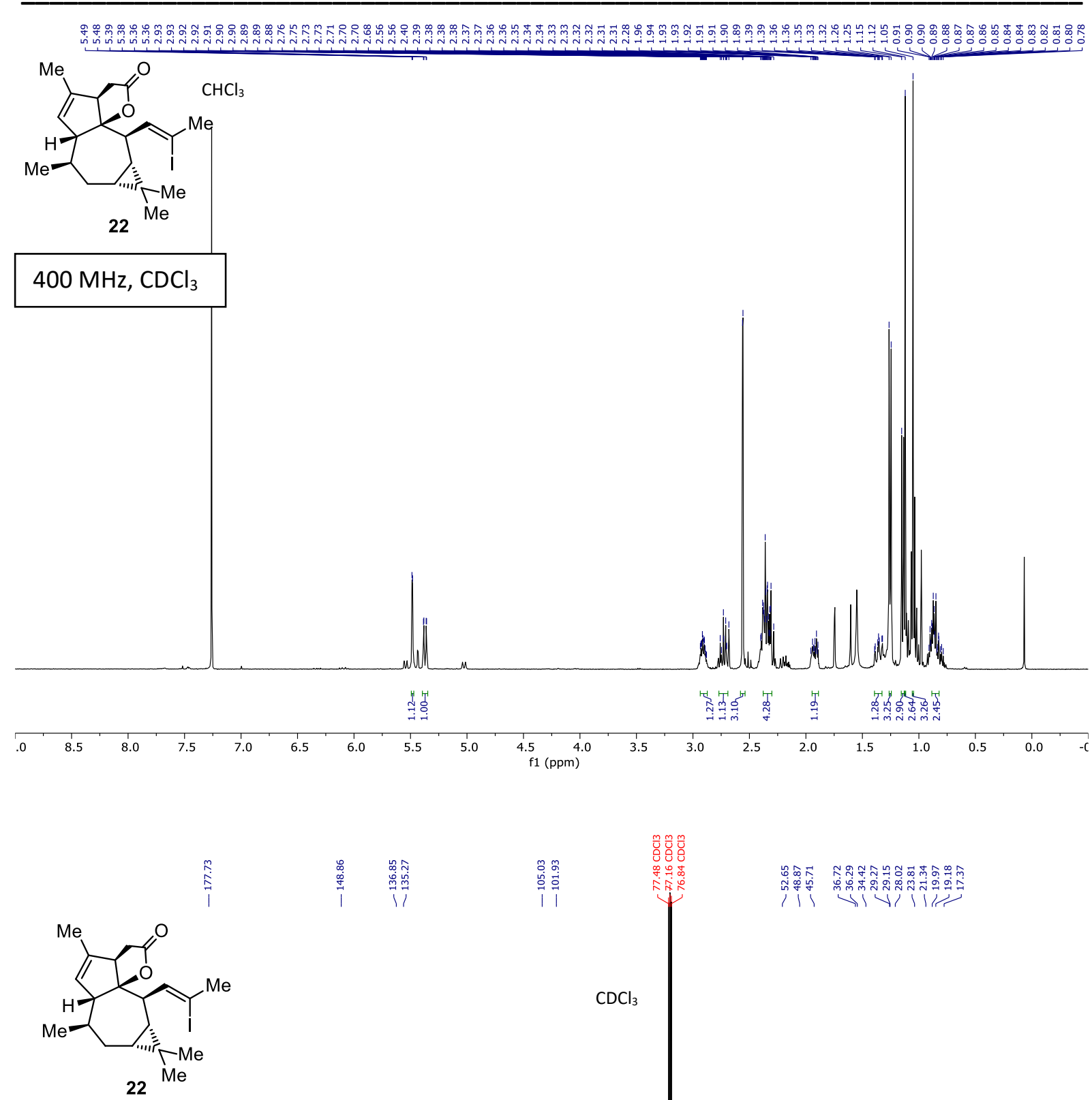

$101 \mathrm{MHz}, \mathrm{CDCl}_{3}$ 


\section{Supporting Information}

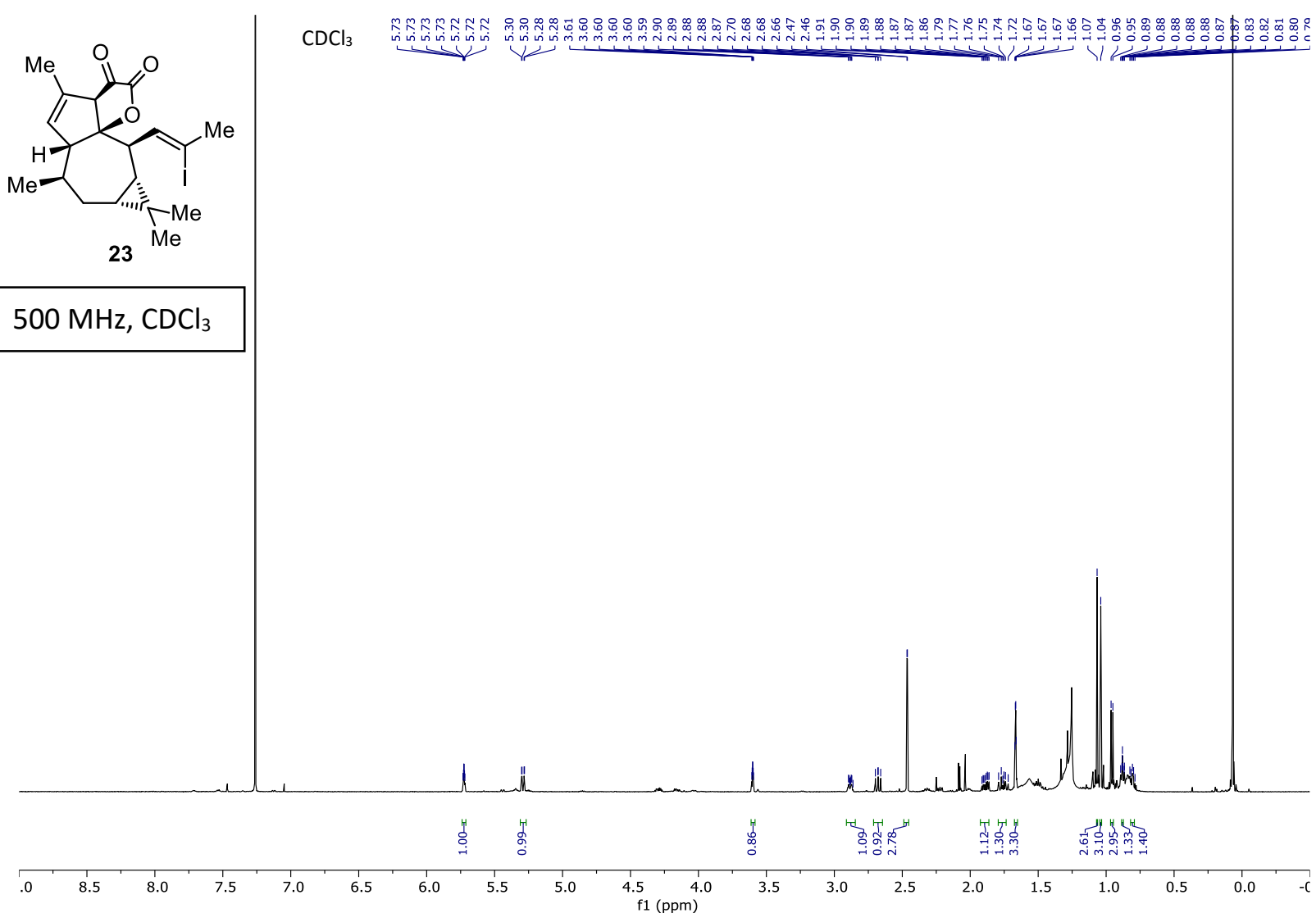

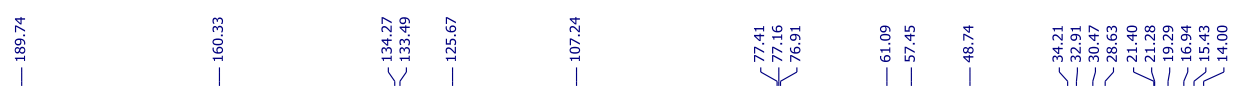

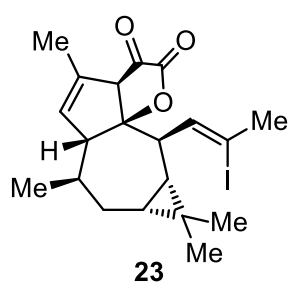

$\mathrm{CDCl}_{3}$

$126 \mathrm{MHz}, \mathrm{CDCl}_{3}$

\begin{tabular}{llllllllllllllllllllllllllllllll}
\hline & 210 & 200 & 190 & 180 & 170 & 160 & 150 & 140 & 130 & 120 & 110 & 100 & 90 & 80 & 70 & 60 & 50 & 40 & 30 & 20 & 10 & 0 & -10
\end{tabular} 


\section{Supporting Information}

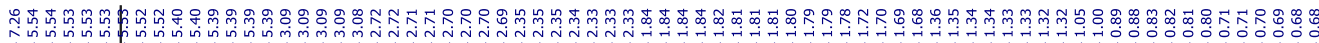

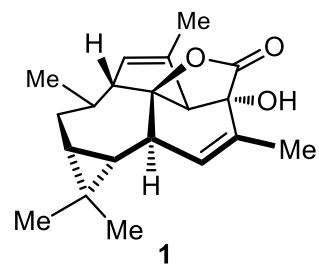

$600 \mathrm{MHz}, \mathrm{CDCl}_{3}$

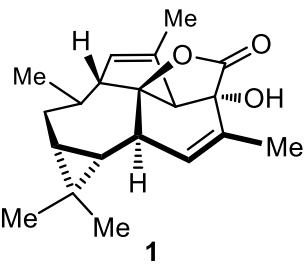

$\mathrm{CDCl}_{3}$

$151 \mathrm{MHz}^{\mathrm{CDCl}_{3}}$

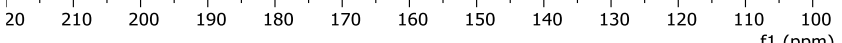

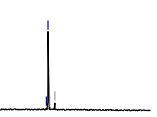


Comparison of natural and synthetic 1.

\begin{tabular}{lll}
\hline $\mathbf{H}$ & Natural $[\mathrm{ppm}]^{5}$ & Synthetic [ppm] \\
\hline $\mathbf{1}$ & 5.53 & 5.53 \\
$\mathbf{2}$ & - & - \\
$\mathbf{3}$ & 3.09 & 3.09 \\
$\mathbf{4}$ & - & - \\
$\mathbf{5}$ & 2.71 & 2.71 \\
$\mathbf{6}$ & 1.34 & 1.34 \\
$\mathbf{7}$ & $1.79,1.68$ & $1.79,1.72-1.68$ \\
$\mathbf{8}$ & 0.68 & 0.69 \\
$\mathbf{9}$ & - & - \\
$\mathbf{1 0}$ & 0.81 & $0.79-0.82$ \\
$\mathbf{1 1}$ & 2.34 & 2.34 \\
$\mathbf{1 2}$ & 5.40 & 5.39 \\
$\mathbf{1 3}$ & - & - \\
$\mathbf{1 4}$ & - & - \\
$\mathbf{1 5}$ & - & - \\
$\mathbf{1 6}$ & 1.84 & 1.81 \\
$\mathbf{1 7}$ & 0.89 & 0.88 \\
$\mathbf{1 8}$ & 1.05 & 1.05 \\
$\mathbf{2 0}$ & 1.00 & 1.00 \\
\hline & 1.81 & 1.84 \\
\hline & & \\
\hline
\end{tabular}


Supporting Information

\begin{tabular}{lll}
\hline $\mathbf{C}$ & Natural $[\mathbf{p p m}]^{5}$ & Synthetic [ppm] \\
\hline $\mathbf{1}$ & 130.6 & 130.6 \\
$\mathbf{2}$ & 137.3 & 137.3 \\
$\mathbf{3}$ & 59.6 & 59.7 \\
$\mathbf{4}$ & 97.3 & 97.3 \\
$\mathbf{5}$ & 54.5 & 54.6 \\
$\mathbf{6}$ & 31.3 & 31.3 \\
$\mathbf{7}$ & 32.7 & 32.7 \\
$\mathbf{8}$ & 20.8 & 20.9 \\
$\mathbf{9}$ & 18.8 & 18.8 \\
$\mathbf{1 0}$ & 26.5 & 26.6 \\
$\mathbf{1 1}$ & 39.1 & 39.1 \\
$\mathbf{1 2}$ & 126.1 & 126.1 \\
$\mathbf{1 3}$ & 139.5 & 139.5 \\
$\mathbf{1 4}$ & 80.4 & 80.4 \\
$\mathbf{1 5}$ & 177.0 & 177.0 \\
$\mathbf{1 6}$ & 16.2 & 16.2 \\
$\mathbf{1 7}$ & 20.9 & 20.9 \\
$\mathbf{1 8}$ & 28.6 & 28.6 \\
$\mathbf{1 9}$ & 15.3 & 15.3 \\
$\mathbf{2 0}$ & 16.6 & 16.6 \\
\hline & & \\
\hline & & \\
\hline
\end{tabular}

\section{IR spectrum of $1:$}

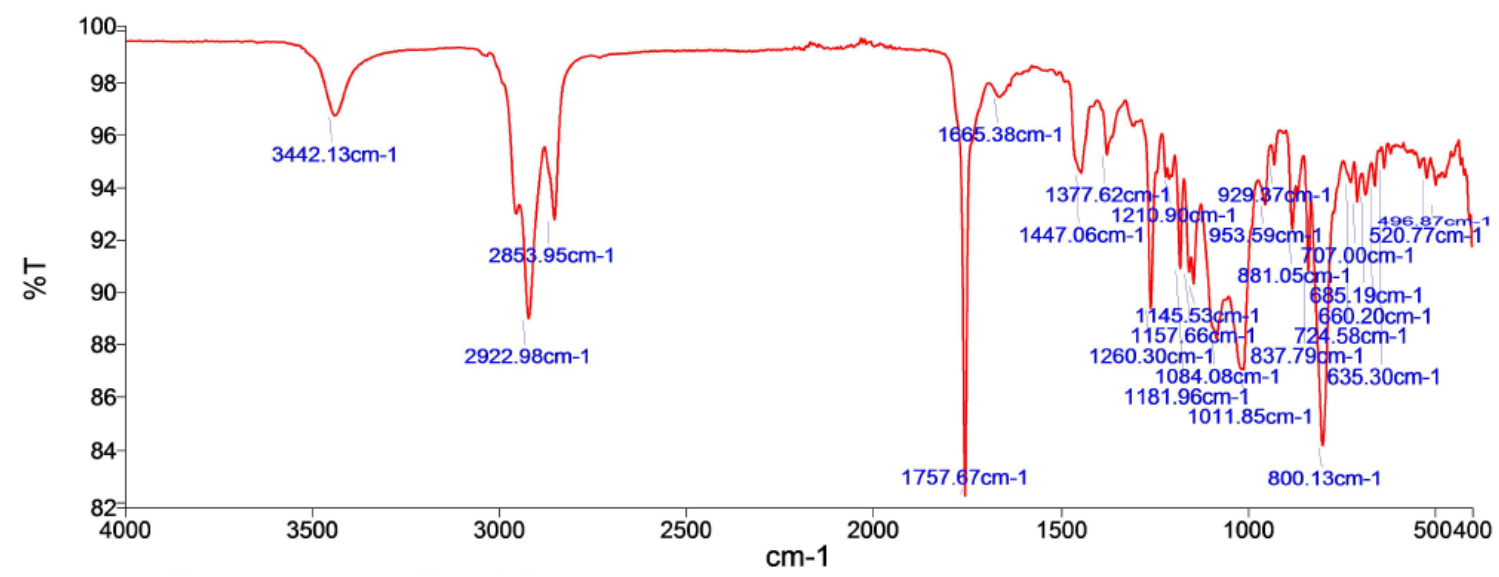




\section{Supporting Information}

\section{Sources}

1. P. Girard; J. L. Namy; H. B. Kagan, Divalent lanthanide derivatives in organic synthesis. 1. Mild preparation of samarium iodide and ytterbium iodide and their use as reducing or coupling agents. $J$ Am Chem Soc 1980, 102 (8), 2693-2698.

2. T. Chen; K.-H. Altmann, Directed Hydrogenations and an Ireland-Claisen Rearrangement Linked to Evans-Tishchenko Chemistry: The Highly Efficient Total Synthesis of the Marine Cyclodepsipeptide Doliculide. Chemistry - A European Journal 2015, 21 (23), 8403-8407.

3. T. Satoh; Y. Kaneko; T. Okuda; S. Uwaya; K. Yamakawa, Studies on the Terpenoids and Related Alicyclic Compounds. XXXV. Studies Directed toward a Total Synthesis of Ingenol Esters : Synthesis of the C/D-Ring Moiety of Ingenol Esters from (+)-3-Carene via Tin (IV) Chloride-Promoted Intramolecular Directed Aldol Reaction. CHEMICAL \& PHARMACEUTICAL BULLETIN 1984, 32 (9), 3452-3460.

4. R. K. Jackson; J. L. Wood, Total Synthesis of ent-Plagiochianin B. Organic Letters 2021.

5. D.-Q. Fei; L.-L. Dong; F.-M. Qi; G.-X. Fan; H.-H. Li; Z.-Y. Li; Z.-X. Zhang, Euphorikanin A, a Diterpenoid Lactone with a Fused 5/6/7/3 Ring System from Euphorbia kansui. Organic Letters 2016, 18 (12), 2844-2847. 